\title{
Constraints on the path-length dependence of jet quenching in nuclear collisions at RHIC and LHC
}

\author{
Barbara Betz $^{a}$ and Miklos Gyulassy ${ }^{b, c, d}$ \\ ${ }^{a}$ Institute for Theoretical Physics, Johann Wolfgang Goethe-University, \\ 60438 Frankfurt am Main, Germany \\ ${ }^{b}$ Department of Physics, Columbia University, \\ New York, 10027, U.S.A. \\ ${ }^{c}$ Nuclear Science Division, Lawrence Berkeley National Laboratory, \\ Berkeley, CA, U.S.A. \\ ${ }^{d}$ Institute for Particle and Nuclear Physics, Wigner RCP, \\ HAS, 1121 Budapest, Hungary \\ E-mail: betz@th.physik.uni-frankfurt.de, gyulassy@phys.columbia.edu
}

ABSTRACT: Recent data on the high- $p_{T}$ pion nuclear modification factor, $R_{A A}\left(p_{T}\right)$, and its elliptic azimuthal asymmetry, $v_{2}\left(p_{T}\right)$, from RHIC/BNL and LHC/CERN are analyzed in terms of a wide class of jet-energy loss models coupled to different $(2+1) \mathrm{d}$ transverse plus Bjorken expanding hydrodynamic fields. We test the consistency of each model by demanding a simultaneous account of the azimuthal, the transverse momentum, and the centrality dependence of the data at both 0.2 and $2.76 \mathrm{ATeV}$ energies. We find a rather broad class of jet-energy independent energy-loss models $d E / d x=\kappa(T) x^{z} T^{2+z} \zeta_{q}$ that, when coupled to bulk constrained temperature fields $T(x, t)$, can account for the current data at the $\chi^{2} /$ d.o.f. $<2$ level with different temperature-dependent jet-medium couplings, $\kappa(T)$, and path-length dependence exponents $0 \leq z \leq 2$. We extend previous studies by including a generic term, $0<\zeta_{q}<2+q$, to test different scenarios of energy-loss fluctuations. While a previously proposed AdS/CFT jet-energy loss model with a temperature-independent jet-medium coupling as well as a near- $T_{c}$ dominated, pQCD-inspired energy-loss scenario are shown to be inconsistent with the LHC data, once the parameters are constrained by fitting to RHIC results, we find several new solutions with a temperature-dependent $\kappa(T)$. We conclude that the current level of statistical and systematic uncertainties of the measured data does not allow a constraint on the path-length exponent $z$ to a range narrower than $[0-2]$.

KeYwords: Jets, Heavy Ion Phenomenology

ARXIV EPRINT: 1404.6378 


\section{Contents}

1 Introduction 1

2 The $(z, c, q)$-classification of $d E / d x$-models 3

$3 \quad$ RHIC and LHC results 4

$\begin{array}{llr}4 & \text { Conclusions } & 17\end{array}$

$\begin{array}{ll}\text { A Jet+bulk models used in the present survey } & 19\end{array}$

\section{Introduction}

Jet-quenching observables [1-6] have been proposed as tomographic probes of the density evolution of quark-gluon plasmas (QGPs) produced in high-energy nuclear collisions. It has been found that the nuclear modification pattern of jet distributions depends on a delicate complex interplay between the details of the jet-medium dynamics, $d E / d x=$ $d E / d x[E(t), \vec{x}(t), T(t)]$, and the evolution of the bulk QGP collective temperature fields, $T(t)=T[\vec{x}(t), t]$.

Below we investigate a wide variety of jet-energy loss models coupled to different QGP temperature fields constrained by bulk observables from state-of-the-art (viscous) $(2+1)$ d hydrodynamic prescriptions [7-15]. We compare the obtained model results to recent data on the nuclear modification factor $R_{A A}\left(p_{T}, \phi, b, \sqrt{s}\right)$ and the high- $p_{T}$ elliptic flow $v_{2}\left(p_{T}, \phi, b, \sqrt{s}\right)[16-22]$, investigating the transverse momentum $p_{T}$, the azimuthal $\phi$, the centrality $b$, and the collision energy $\sqrt{s}$ dependence of the data measured at both the Relativistic Heavy Ion Collider (RHIC) and the Large Hadron Collider (LHC) with a special focus on the robustness of results for high- $p_{T}>7-10 \mathrm{GeV}$ hadron fragments from jets.

In particular, we study results based on a class of jet-energy loss models that can be parametrized as $d E / d x=\kappa(T) E^{a} x^{z} T^{c=2+z} \zeta_{q}$. The jet-energy dependence, the path-length dependence, and the temperature dependence are characterized by the exponents $(a, z, c)$. The above form allows for different assumptions of the distribution of the relative energyloss fluctuations through a multiplicative factor $\zeta_{q}$, specifying a parameter $q$ as discussed in the text below, and rendering the $(a, z, c, q)$-prescription. This class of models includes perturbative QCD (pQCD) based models with exponents $(0,0,2, q)$ and $(0,1,3, q)$, conformal AdS holography models with non-linear path length $(0,2,4, q)$, and a phenomenological model assuming an enhancement of the jet-energy loss near $T_{c} \approx 170 \mathrm{MeV}$ as in ref. [23-25], here referred to as the SLTc model with $(0,1,3, q)$.

Since we find (see tables 2 and 3 in the appendix) that all these models have problems to simultaneously account for the various data measured both at RHIC and LHC, once the 
parameters are fixed at RHIC energies, we consider different deformations of those models by varying the assumed temperature dependence of the jet-medium coupling, $\kappa(T)$. We confirm previous results pointing to the need to reduce of the jet-medium coupling from LHC to RHIC [26-38], with a jet-medium coupling $\kappa_{\mathrm{LHC}} \approx 0.5 \kappa_{\mathrm{RHIC}}$ that negates most of the increase of the jet-energy loss as expected from the factor of $\sim 2$ increase of the QGP density at LHC relative to RHIC.

However, even after that reduction is taken into account to describe the $R_{A A}$ at in the $p_{T} \sim 10 \mathrm{GeV}$ range, its elliptic azimuthal moment, $v_{2}$, is still found to be significantly underestimated by most models, especially at the LHC, in line with various pQCD-based models (AMY, HT, ASW, Molnar, CUJET2.0) [16, 39-41] that are about a factor of $\sim 2$ below the measured data and might depend on the running of the coupling constant with both, the temperature and the scale $\alpha_{\text {eff }}(Q, T)[39,42]$. We therefore further explore deformations of the models that could help to resolve this "high- $p_{T} v_{2}$-problem".

We find that the pQCD-based models describing a vacuum running coupling with radiative energy-loss, $(0,1,3, q)$, require only a modest $10-15 \%$ difference between the pathaveraged coupling in- and out-of reaction plane. For AdS-like models, $(0,2,4, q)$, strong non-conformal temperature variations are required to bring those predictions closer to the combined RHIC and LHC data. Finally, a more radical deformation of the SLTc model with an exponential suppression of high-temperature jet-energy loss is reported that appears to be consistent within the present experimental and theoretical errors at RHIC and LHC.

The present work was motivated in part by a recent PHENIX study [16] suggesting the tentative conclusion that an AdS/CFT-motivated jet-energy loss prescription with $d E / d x \sim \kappa x^{2} T^{4}$ [43-47] coupled to a particular hydrodynamic background [43, 44] is more consistent with the observed azimuthal asymmetries than results based on pQCD radiative $(0,1,3, q)$ and elastic $(0,0,2, q)$ energy-loss models at RHIC energies used by AMY, HT, and ASW cited in ref. [16].

Here, we test the consistency of those models, parametrized by $(a, z, c, q)$, with the observed $\sqrt{s}$ dependence between RHIC and LHC. As mentioned above, LHC jets probe the QGP phase of matter with up to an order of magnitude higher $p_{T}$-range under conditions where the QGP density $\propto T^{3}$ is more than doubled relative to RHIC. In addition, cross comparisons between RHIC and LHC are useful as the initial invariant jet-production distributions at midrapidity, $y=0$, denoted here as $g_{r}\left(p_{T}\right)=d N_{r}^{j e t} / d y d^{2} p_{T}$ for $r=q, g$ jets, changes by orders of magnitude from RHIC to LHC. Therefore, demanding a simultaneous description of RHIC and LHC data provides the most stringent test so far of the consistency and quantitative predictive power of proposed dynamical models of jet-energy loss and of the space-time evolution of the bulk QGP density produced in ultra-relativistic heavyion collisions.

Both, the magnitude and the azimuthal dependence of jet quenching in non-central collisions are conveniently studied via the nuclear modification factors in- and out-of-plane $R_{A A}^{\text {in/out }}=R_{A A}\left(1 \pm 2 v_{2}\right)[16,43,44]$, giving simultaneous access to both the nuclear modification factor and the high- $p_{T}$ elliptic flow. These observables are sensitive to all details of the jet energy, the path length, and the temperature dependence of jet-energy loss models (see, e.g. refs. [14, 15, 40, 41, 48-51]). In particular, they depend on the details of the QGP 
transverse as well as longitudinal expansion [7-13, 52], as emphasized by Renk [48] and Molnar [40, 41].

The jet-medium coupling $\kappa$ used is constrained for each model by fitting to a single reference point at $p_{T}=7.5 \mathrm{GeV}$ in most central $\mathrm{Au}+\mathrm{Au}$ at $\sqrt{s}=200 \mathrm{AGeV}$ RHIC energies with the value of $R_{A A}\left(p_{T}\right)=0.2$, as in refs. [26-28, 43, 44, 53]. However, results are found to be qualitatively insensitive to the particular choice of the $p_{T}$-reference point.

We generalize our previous work [26-28] by including more realistic energy-loss fluctuations to our model and discussing the implications of the high- $p_{T} v_{2}$-problem on the jet-medium coupling.

\section{The $(z, c, q)$-classification of $d E / d x$-models}

In order to interpolate between the different jet-energy loss prescriptions, we utilize a convenient parametric model introduced in refs. [26-31] that originally characterized the jet-energy loss by three exponents $(a, z, c)$ controlling the jet energy $a$, path length $z$, and thermal-field dependence $c$. Here, we allow for additional energy-loss fluctuations $\zeta_{q}$, discussed below, and the possibility that the jet-medium coupling, $\kappa(T)$, may depend non-monotonically on the local temperature field,

$$
\frac{d E}{d x}=\frac{d P}{d \tau}\left(\vec{x}_{0}, \phi, \tau\right)=-\kappa(T) P^{a}(\tau) \tau^{z} T^{c} \zeta_{q}
$$

where $\kappa(T)=C_{r} \kappa^{\prime}(T)$ and $T=T\left[\vec{x}(\tau)=\vec{x}_{0}+\left(\tau-\tau_{0}\right) \hat{n}(\phi), \tau\right]$ describes the local temperature along the jet path at time $\tau$ for a jet initially produced time $\tau_{0}$. The jets are distributed according to a transverse initial profile specified by the bulk QGP flow fields given by three variants of transverse plus Bjorken $(2+1)$ d expansion: (1) VISH2+1 [7-10], (2) viscous RL hydro [12, 13], and (3) a $v_{\perp}=0.6$ blast wave flow [14, 15] assuming radial dilation of the initial transverse profile: $\rho(x, y, \tau)=\rho_{0}[x / r(\tau), y / r(\tau)]\left[\tau_{0} / \tau r^{2}(\tau)\right]$ with $r(\tau)=\left(1+v_{\perp}^{2} \tau^{2} / R^{2}\right)^{1 / 2}$. Here, $R$ denotes the initial root mean square radius. For dimensionless couplings $\kappa, c=2+z-a$. In eq. (2.1), $C_{r}=1\left(\frac{9}{4}\right)$ describes quark (gluon) jets. For jets of type $r=q, g$ produced with an invariant transverse momentum distribution $g_{r}\left(P_{0}\right)$ taken from refs. [26-31], the nuclear modification factor is given by

$$
R_{A A}^{r}\left(P_{f}, \phi\right)=\frac{\left\langle g_{r}\left[P_{0}^{r}\left(P_{f}, \phi\right)\right]\right\rangle}{g_{r}\left(P_{f}\right)} \frac{d P_{0}^{2}}{d P_{f}^{2}}
$$

The ensemble average is taken over initial jet-production points and initial $\sqrt{s}$-dependent initial jet energies $P_{0}$, as well as parameters controlling the energy loss, geometry, and temperature background fields.

For a given member of the jet ensemble, the average initial jet energy $P_{0}^{r}$, is related to the final quenched jet energy (prior to hadronization) $P_{f}$ via a path integral that we here assume to be an Eikonal straight line in azimuthal direction $\phi$. For a particular jet flavor $r$, the average initial jet energy is

$$
P_{0}^{r}\left(P_{f}, \phi\right)=\left[P_{f}^{1-a}+\zeta_{q} \int_{\tau_{0}}^{\tau_{f}} K_{r}(T) \tau^{z} T^{c}\left[\vec{x}_{\perp}(\tau), \tau\right] d \tau\right]^{\frac{1}{1-a}}
$$


with the effective jet-medium coupling $K_{r}(T)=(1-a) C_{r} \kappa(T)$. Eq. (2.3) illustrates the competing effects due to the intrinsic $d E / d x \propto E^{a} x^{z} T^{c}$ energy-loss details, the impact of local hydrodynamic temperature fields, and a possible non-monotonic jet-medium coupling $\kappa(T[\vec{x}(t), t])$ along its path. We checked numerically that local transverse flow-field effects introduced in ref. [54] do not significantly influence the results based on eq. (2.3).

With eq. (2.3) we have further generalized the class of $(a, z, c)$-models to $(a, z, c, q)$ models that include the possibility of skewed jet-energy loss fluctuations about its pathaveraged mean using a scaling factor $0<\zeta_{q}<q+2$ and being distributed according to

$$
f_{q}\left(\zeta_{q}\right)=\frac{(1+q)}{(q+2)^{1+q}}\left(q+2-\zeta_{q}\right)^{q}
$$

with a root-mean square of $\left\langle\left(\zeta_{q}-1\right)^{2}\right\rangle=(q+1) /(q+2)$. This class of skewed distributions is controlled by a parameter $q>-1$ with unit mean, $\left\langle\zeta_{q}\right\rangle=1$. It conveniently interpolates between non-fluctuating $\left(q=-1, \zeta_{-1}=1\right)$, uniform Dirac $\delta\left(1-\zeta_{-1}\right)=\lim _{q \rightarrow-1^{+}} f_{q}\left(\zeta_{-1}\right)$ distributions between $0<\zeta_{-1}<1$, and increasingly skewed distributions towards small $\zeta_{q}<1$ for $q>-1$ similar to pQCD based models, see e.g., refs. [1-5, 29-31, 55, 56]. Note that current non-perturbative AdS and the originally proposed SLTc models [23-25] do not include fluctuations of the jet-energy loss about its path average and thus correspond to the $q=-1, \zeta_{-1}=1$ limit of eq. (2.4).

Including the energy-loss fluctuations specified by eqs. (2.3) and (2.4) into eq. (2.1) thus conveniently classifies jet-medium models labelled by $(a, z, c, q)$ to differentiate between a much broader class of jet-energy loss models than reported in ref. [26-28]. By varying these four model space parameters we aim to quantify the exponents and to identify which combinations of jet-energy loss and bulk QGP evolution models can be ruled out by the current RHIC and LHC data.

We limit the study to the special cases $a=0, z=[0,1,2], c=2+z$, and $q=[-1,0,1]$, and hence the $(z, c, q)$-model. Our restriction to $a=0$ and thus jet-energy independent energy-loss models is motivated by earlier results reported in ref. [26-28] showing that the slope of $R_{\mathrm{PbPb}}\left(10<p_{T}<40 \mathrm{GeV}, 0-10 \%\right.$ centrality, $\left.\sqrt{s}=2.76 \mathrm{ATeV}\right)$ strongly disfavors models with $a>1 / 3$.

In particular, we investigate a pQCD-like radiative energy loss QCDrad with $d E / d x=$ $\kappa E^{0} \tau^{1} T^{3} \zeta_{q}$, a pQCD-like elastic energy loss QCDel with $d E / d x=\kappa E^{0} \tau^{0} T^{2} \zeta_{q}$, an AdS/CFT-inspired scenario with $d E / d x=\kappa E^{0} \tau^{2} T^{4} \zeta_{q}$, and a SLTc model with $d E / d x=$ $\kappa(T) E^{0} \tau^{1} T^{3} \zeta_{q}$ that has a coupling constant with a constant, non-negligible value for large temperatures that is enhanced around $T \sim T_{c} \approx 170 \mathrm{MeV}$ [23-25]. Besides that, we also study two models with a jet-medium coupling that either depends on the azimuth [39] or drops exponentially for large temperates. We consider a weaker $\kappa_{\mathrm{LHC}}<\kappa_{\mathrm{RHIC}}$ coupling at LHC energies for the pQCD-like jet-energy loss prescriptions [26-36, 39].

\section{$3 \quad$ RHIC and LHC results}

Figure 1 shows the most central (black lines) and more peripheral pion nuclear modification factors in- and out-of-plane (red and blue lines) for the first four model scenarios introduced 

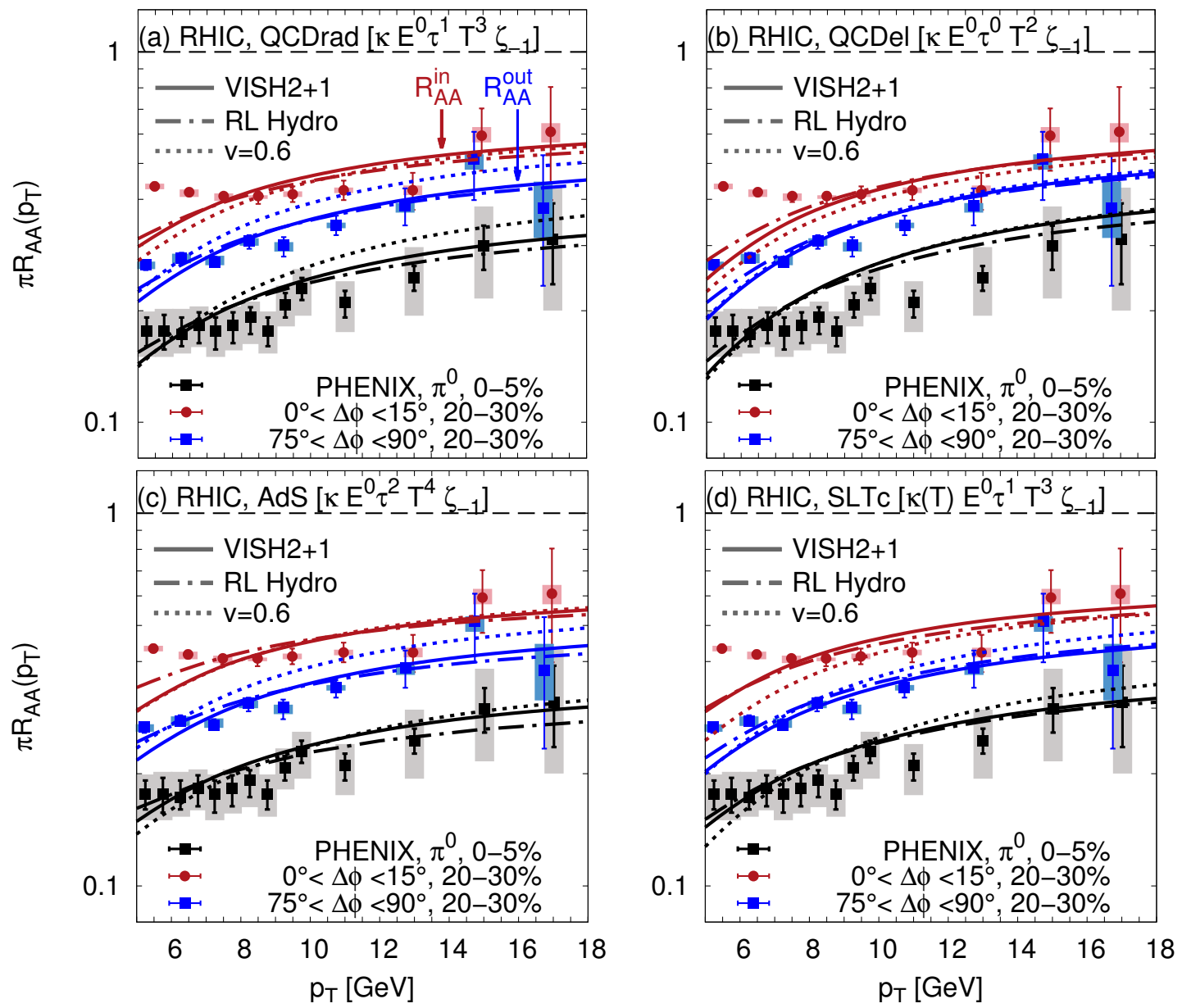

Figure 1. Azimuthal jet tomography at RHIC. Panels (a-d) show PHENIX 200AGeV Au+Au data [16] on the $\pi^{0}$ nuclear modification factors for most central 0-5\% collisions (black lines) as well as their in- and out-of-plane contributions for $20-30 \%$ centralities (red and blue lines), compared to predictions based on $d E / d x=\kappa E^{a=0} x^{z} T^{c=2+z} \zeta_{-1}$ [26-31] without energy loss fluctuations, i.e., $\zeta_{-1}=1$. Panel (a), labelled QCDrad $(z, c, q)=(1,3,-1)$, corresponds to a radiative pQCDenergy loss including running-coupling effects [26-28, 32-36, 39], panel (b), denoted QCDel with $(z, c, q)=(0,2,-1)$, describes an elastic jet-energy scenario [29-31, 55, 56], panel (c), marked as AdS with $(z, c, q)=(2,4,-1)$, simulates an idealized conformal falling string energy loss $[45,58-$ 60], and panel (d), indicated as SLTc with $(z, c, q)=(1,3,-1)$ and $\kappa\left(T_{c}\right)=3 \kappa(\infty)$, simulates a $T_{c}$-dominated energy-loss model proposed in ref. [23-25]. For each model, the quenching pattern is computed using three different bulk QGP fluid-temperature fields: ideal $(\eta / s=0) \mathrm{VISH} 2+1[7,8]$ (solid), viscous $(\eta / s=0.08)$ RL Hydro $[12,13]$ (dashed-dotted), and a simple $v_{\perp}=0.6$ transverse blast wave model $[14,15]$ (dotted).

above in panels (a) to (d) not considering energy-loss fluctuations, i.e. $\zeta_{-1}=1$, for the three $(2+1)$ d flow background fields of ideal VISH2+1 (solid) [7, 8], viscous RL Hydro (dasheddotted) [12, 13], and the $v_{\perp}=0.6$ blast wave flow [14, 15]. Please note that in refs. [32, 33] the opacity integral of eq. (2.3) was evaluated taking only Bjorken expansion with a $v_{\perp}=0$ into account. 
The most striking result in figure 1 is that in contrast to the (AMY, HT, and ASW) pQCD models [43, 44] shown in ref. [16], all models combined with either ideal VISH2+1 or viscous RL Hydro transverse flow fields agree within present errors with the measured RHIC data in the high- $p_{T}>7 \mathrm{GeV}$ region. Only the QCDel model seems to be disfavored as compared to the other scenarios. We checked (not shown) that the results of viscous VISH2 +1 [11] background fields vary by less than $5 \%$. However, the $v_{\perp}=0.6$ transverse blast wave background leads, as in ref. $[14,15]$ with $v_{\perp}=0$, to an in/out asymmetry with a factor of $\sim$ two below the recent PHENIX data [16]. Ref. [40, 41] also reports that the GLV energy-loss [1-5] evaluated in the MPC parton cascade background under-predicts the high$p_{T}$ elliptic asymmetry observed at RHIC. This result [40, 41] was another major motivation for the present work, as well as the detailed investigation provided by CUJET2.0 [39].

The differences between the models shown in figure 1 and the results reported in refs. [16] are due to various combined effects of the jet-energy loss and the bulk QGP flow. The flow fields [43, 44] considered for the study in ref. [16] were computed with an ideal (non-dissipative) hydrodynamic code assuming a Bag model first order-phase transition with a speed of sound vanishing over a wide energy-density range. Here, however, the VISH2+1 results used in figure 1 utilizes a smoothed (SM-EOS Q) equation of state (EoS) and the viscous RL Hydro employs a realistic continuous crossover transition EoS.

Besides that, the results shown in figure 1 do not include jet-energy loss fluctuations as deduced in eq. (2.3) that are intrinsic to the models reported in refs. [39-41]. This effect is, however, included in figure 2 for the three scenarios of QCDrad, QCDel, and AdS, comparing the non-fluctuating case of $q=-1$ to two different energy-loss fluctuations given by $q=0,1$. For reasons of clarity and to better compare to results of CUJET2.0 [39], we restricted this comparison to the VISH2 +1 background fields $[9,10]$ and omitted the SLTc model as the effects of a fluctuating jet-energy loss for a model with $d E / d x \sim E^{0} \tau^{1} T^{3} \zeta_{q}$ is already illustrated by the QCDrad scenario shown in figure 2(a). Please note that the detailed study of ref. [39] demonstrated that the GLV energy-loss considered in both refs. [39-41] may account for the $v_{2}$ data at RHIC for an $\alpha_{\max }=0.26-0.28$. The differences in the results of ref. [39] and [40,41] can only be explained in the details of the (running) coupling constant as well as the background medium considered.

Including jet-energy loss fluctuations, both the QCDrad and the AdS model depicted in figure 2 describe the measured data, while the QCDel scenario is again disfavored. Figure 3 clearly demonstrates that the $q=0$ case reproduces the nuclear modification factor in- and out-of plane with a $\chi^{2} /$ d.o.f. $\leq 1.5$ if a $p_{T}$-range of $8.5<p_{T}<13 \mathrm{GeV}$ is considered. For a wider $p_{T}$-range, even the non-fluctuating $q=-1$ energy loss leads to a decent description with a $\chi^{2} /$ d.o.f. $<2$. Please note that the applicability of our model is limited below $p_{T}=7.5 \mathrm{GeV}$.

Thus, comparing the various models at RHIC energies only allows the conclusion that the QCDrad scenario with and without jet-energy loss fluctuations as well as the AdS scenario and the SLTc model are possible candidates to describe the nuclear modification factor and the high- $p_{T}$ elliptic flow while the QCDel scenario seems to be disfavored.

Given this result as well as the difficulty of untangling the effect of the jet-energy loss and QGP flow fields at one particular collision energy $\sqrt{s}$ lead us to consider the higher 


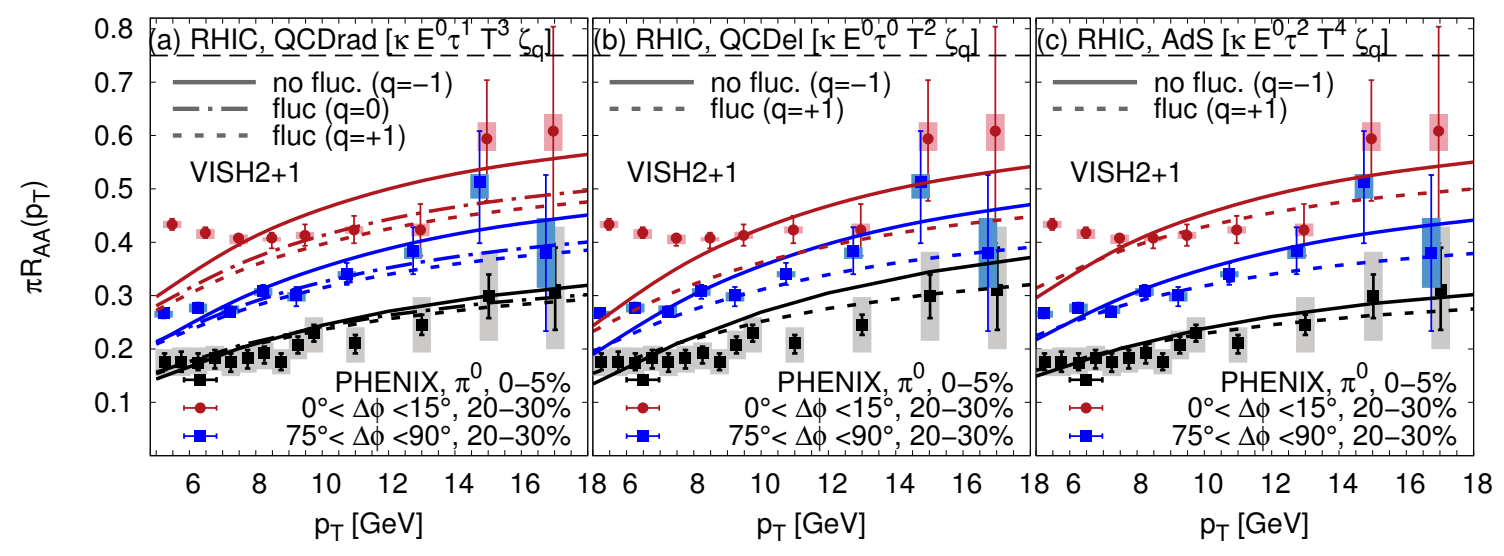

Figure 2. Azimuthal jet tomography at RHIC, comparing predictions based on $d E / d x=$ $\kappa E^{a=0} x^{z} T^{c=2+z} \zeta_{q}[26-31]$ with and without energy fluctuations to PHENIX $200 \mathrm{AGeV} \mathrm{Au}+\mathrm{Au}$ data [16] on $\pi^{0}$ nuclear modification factors for most central 0-5\% collisions and their in- and out-of-plane contributions for 20-30\% centralities. Scenarios (a)-(c) are the same as in figure 1, computed for the ideal VISH2+1 bulk QGP fluid field [7, 8].

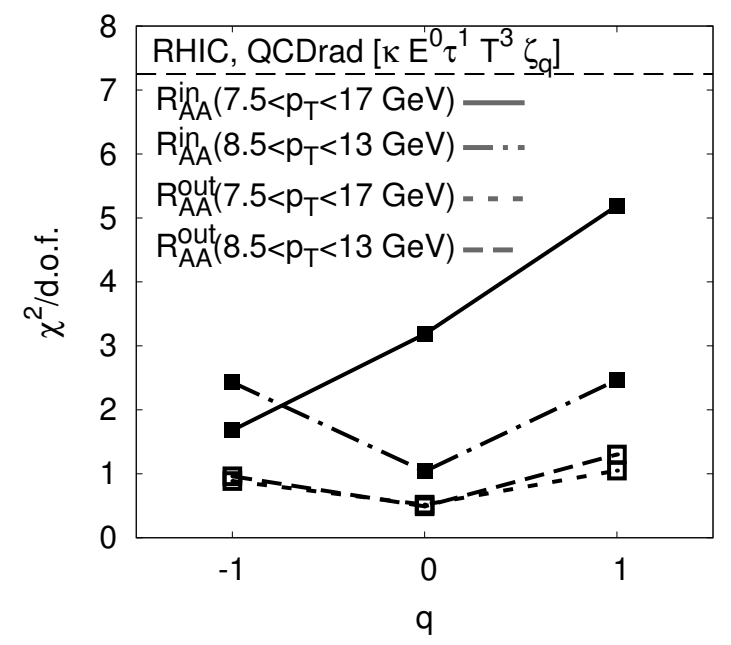

Figure 3. The $\chi^{2} /$ d.o.f. for the QCDrad scenario shown in figure 2 (a) as a function of the three energy-loss fluctuation distributions $q=[-1,0,1]$ considering VISH $2+1$ background fields $[7,8]$. The different $p_{T}$-cuts demonstrate the sensitivity of a $\chi^{2}$-test on the $p_{T}$-range considered.

discriminating power afforded by exploiting the dependence of the $R_{A A}$ and the high- $p_{T}$ elliptic flow on the collision energy in the range of $0.2-2.76 \mathrm{ATeV}$.

We will start the discussion of the LHC results with the pQCD-inspired scenarios QCDrad and QCDel in figures 4-8, while figures 9 and 10 consider the AdS-inspired scenario and the SLTc model. Figures 11-13 then deepen the discussion of a temperature-dependent jet-medium coupling $\kappa(T)$.

Figure 4 depicts the central (black) and more peripheral (red) pion nuclear modification factors as measured by ALICE (dots) [17] and CMS (squares) [19] in the left panels as well as the high- $p_{T}$ elliptic flow as measured by ALICE (filled dots) [18], ATLAS (open 

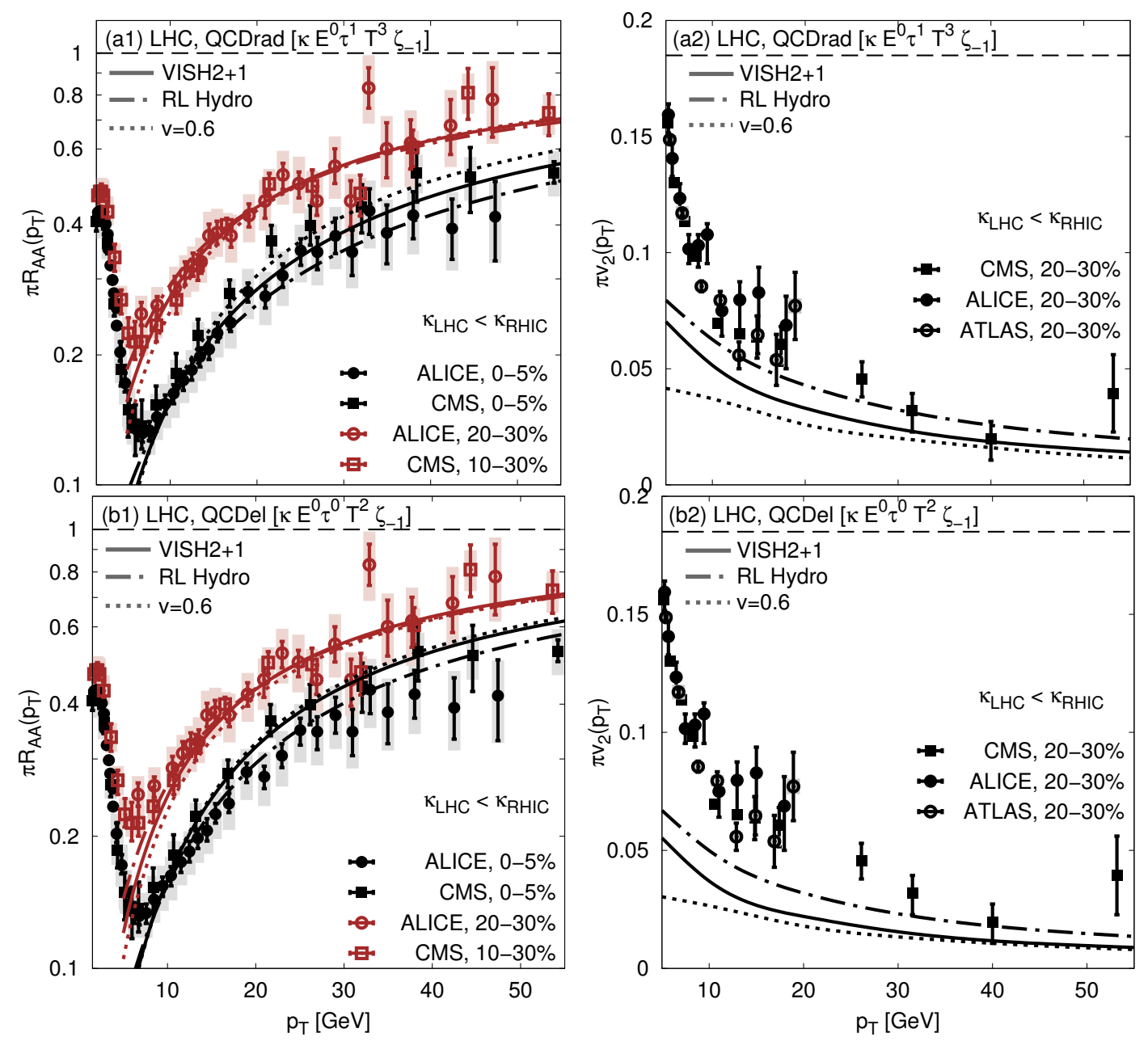

Figure 4. Azimuthal jet tomography at the LHC [17-22]. Panels (a1) and (b1) show the measured data for the pion nuclear modification factor $R_{A A}$ from ALICE [17] and CMS [19] for most central and more peripheral collisions, while panels (a2) and (b2) depict the high- $p_{T}$ elliptic flow as extracted from ALICE [18], CMS [20], and ATLAS [21, 22]. The model calculations are done without energy-loss fluctuations $\left(\zeta_{-1}=1\right)$ to mimic a radiative QCD (QCDrad, upper panel) and an elastic QCD (QCDel, lower panel) energy loss using bulk QGP flow fields at LHC energies from viscous $\eta / s=0.08 \mathrm{VISH} 2+1[9,10]$ (solid), viscous $\eta / s=0.08$ RL Hydro $[12,13]$ (dashed-dotted), and the $v_{\perp}=0.6$ blast wave model $[14,15]$ (dotted). The jet-medium coupling $\kappa_{L H C}$ is reduced relative to RHIC to simulate the running QCD coupling [32-36, 42].

dots) [21, 22], and CMS (squares) [20] in the right panels. Those measured data are compared to the results of the QCDrad (upper panels) and QCDel (lower panels) scenarios for the three different background fields of viscous $(\eta / s=0.08)$ VISH $2+1[9,10]$ (solid), viscous $(\eta / s=0.08)$ RL Hydro $[12,13]$ (dashed-dotted), and the $v_{\perp}=0.6$ blast wave model $[14,15]$ (dotted) without jet-energy loss fluctuations. The jet-medium coupling constant $\kappa$ is lowered to $\sim(40-50) \%$ as compared to RHIC energies to account for running-coupling effects [26-33, 39] explaining the "surprising transparency" [29-31] of the LHC QGP (see also table 2). 

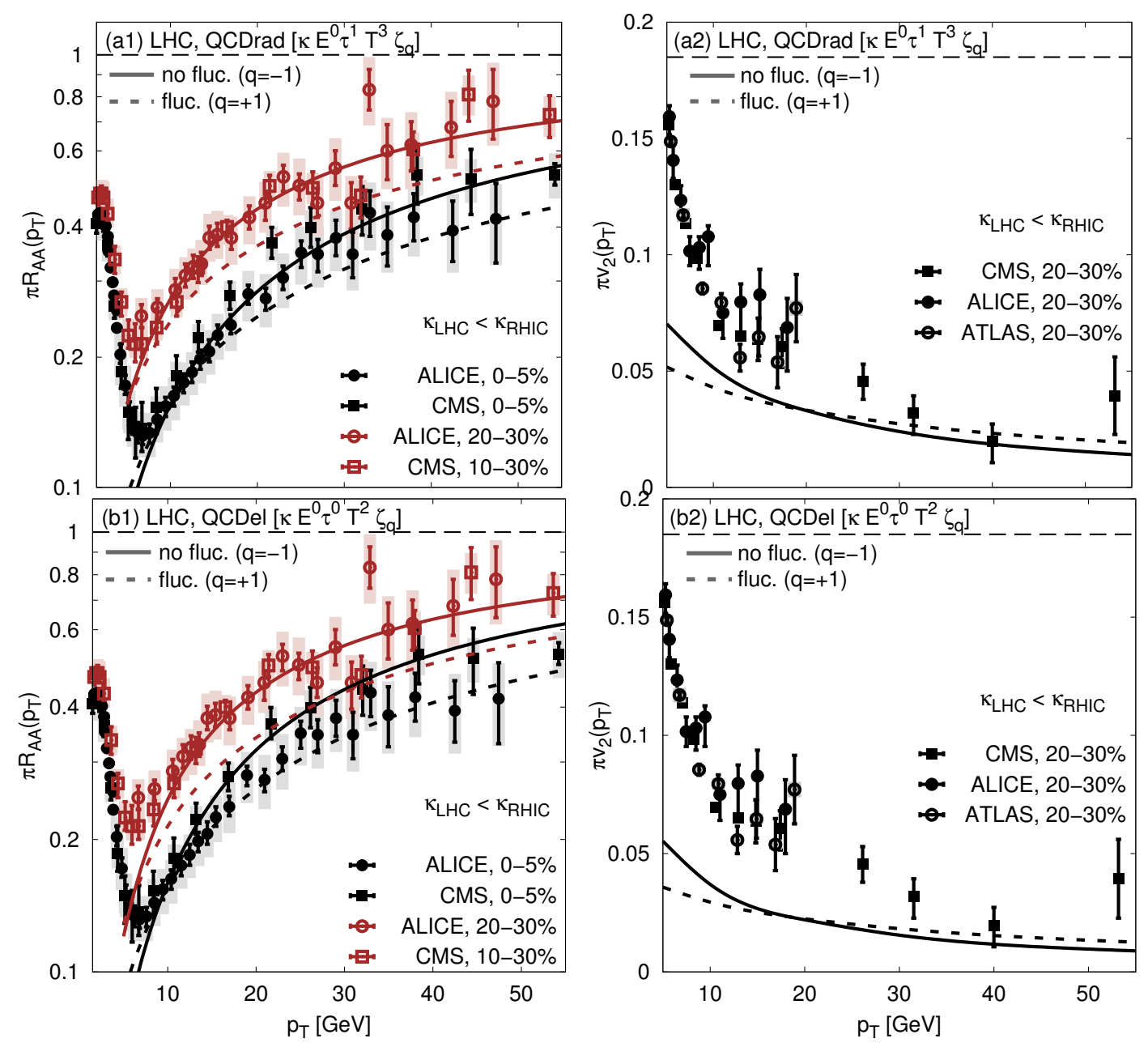

Figure 5. Azimuthal jet tomography at the LHC [17-22] as in figure 4. The model calculations are done for a reduced $\kappa$ value as compared to RHIC, comparing a fluctuating energy-loss scenario (dashed) to the non-fluctuating case (solid) using bulk QGP flow fields at LHC energies from viscous $\eta / s=0.08 \mathrm{VISH} 2+1[9,10]$.

A reduction of the effective jet-medium coupling with $\sqrt{s}$ is natural $[32-36,42]$ in perturbative QCD based jet-energy loss due to vacuum running of both radiative emission and elastic scattering couplings, $\kappa_{Q C D} \propto \alpha_{s}\left(k_{\perp}^{2} /[x(1-x)]\right) \alpha_{s}^{2}\left(Q^{2}\right)$, as a function of the radiated gluon momentum fraction $x$, the gluon transverse momentum $k_{\perp}$, and the medium momentum transfers $Q$. Lattice QCD [42] predicts that $\alpha_{\text {effective }}(Q, T)=\alpha_{\text {eff }}(Q, T)$ runs also with the temperature scale.

Figure 4 demonstrates that the QCDrad scenario with a $d E / d x=\kappa E^{0} \tau^{1} T^{3} \zeta_{-1}$ reproduces both the nuclear modification factors for most central and more peripheral collisions, as well as the high- $p_{T}$ elliptic flow for either the viscous VISH2+1 or the RL Hydro background fields given the uncertainties of the bulk space-time evolution expressed, amongst others, by the initial conditions, the initial time $\tau_{0}$, the shear viscosity over entropy ratio $\eta / s$, and the the freeze-out time $T_{f}$. Neglecting these uncertainties, as done in figure 7 below, the high- $p_{T}$ elliptic flow for VISH $2+1$ is not well described. Please note that we 

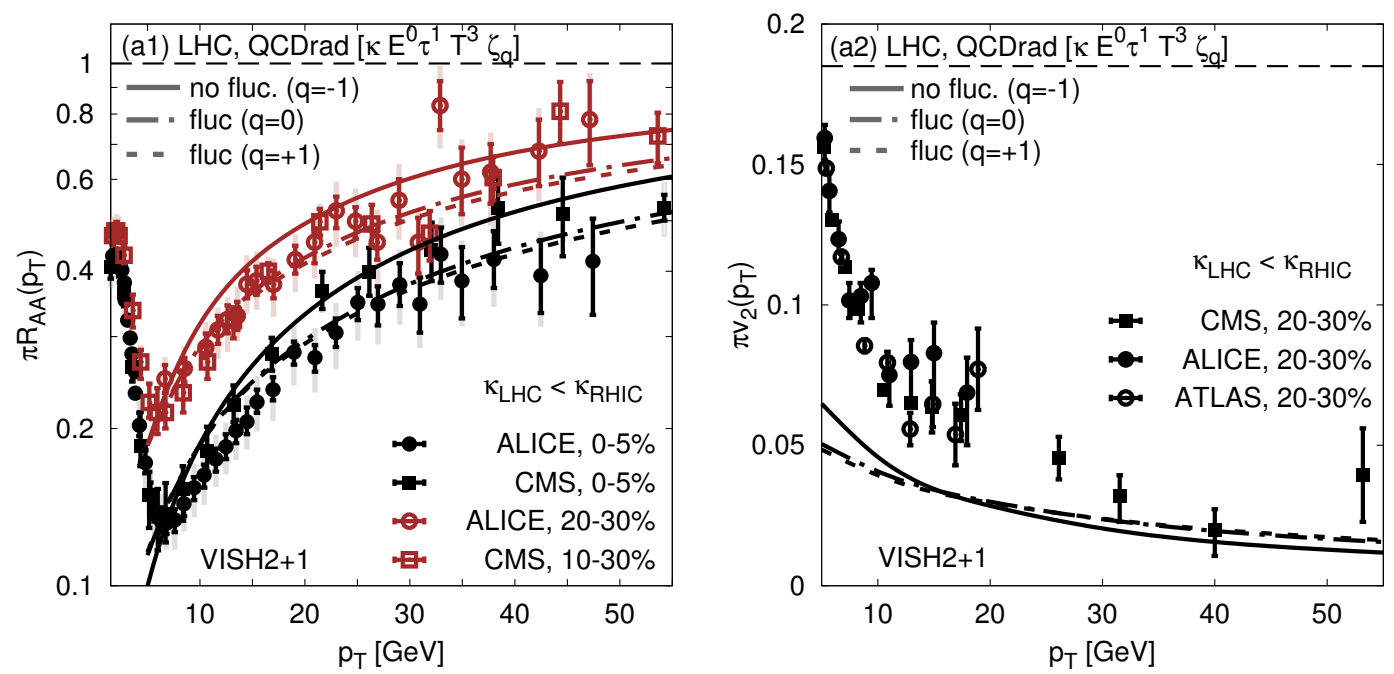

Figure 6. Azimuthal jet tomography at the LHC [17-22] as in figure 5 (a1) and (a2) considering both fluctuating and non-fluctuating jet-energy loss scenarios $(q=-1,0,1)$ but assuming that $R_{A A}\left(p_{T}=10 \mathrm{GeV}\right)=0.186$ instead of $R_{A A}\left(p_{T}=10 \mathrm{GeV}\right)=0.155$ as in figure 5 .

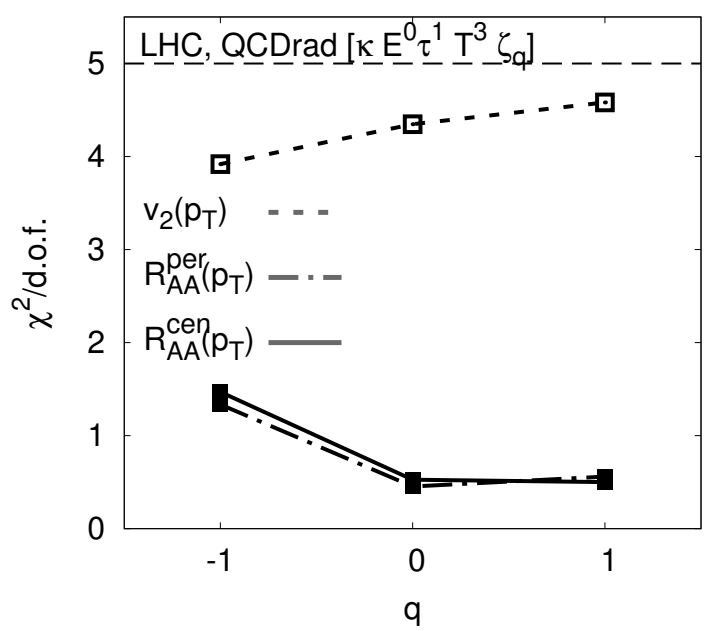

Figure 7. The $\chi^{2} /$ d.o.f. for the QCDrad scenario considering VISH $2+1[9,10]$ background fields shown in figure 6 as a function of the three energy-loss fluctuation distributions $\zeta_{q}=[-1,0,1]$. For the ALICE data, a $p_{T}$-range of $10<p_{T}<48 \mathrm{GeV}$ is considered for the pion nuclear modification factor and $10<p_{T}<20 \mathrm{GeV}$ for the high- $p_{T}$ elliptic flow, while for the CMS data the $p_{T}$-cut of $10<p_{T}<54 \mathrm{GeV}$ holds both for the nuclear modification factor and the high- $p_{T}$ elliptic flow.

consider the uncertainties in the hydrodynamic prescriptions important and thus conclude that the QCDrad scenario without jet-energy loss fluctuations provides a description of the measured data. The $v_{\perp}=0.6$ blast wave model, however, again fails to describe the measured data. Moreover, the QCDrad prescription seems to be favored over the QCDel scenario as for the latter one the high- $p_{T}$ elliptic flow is by $\sim 2$ reduced as compared to the QCDrad results. 

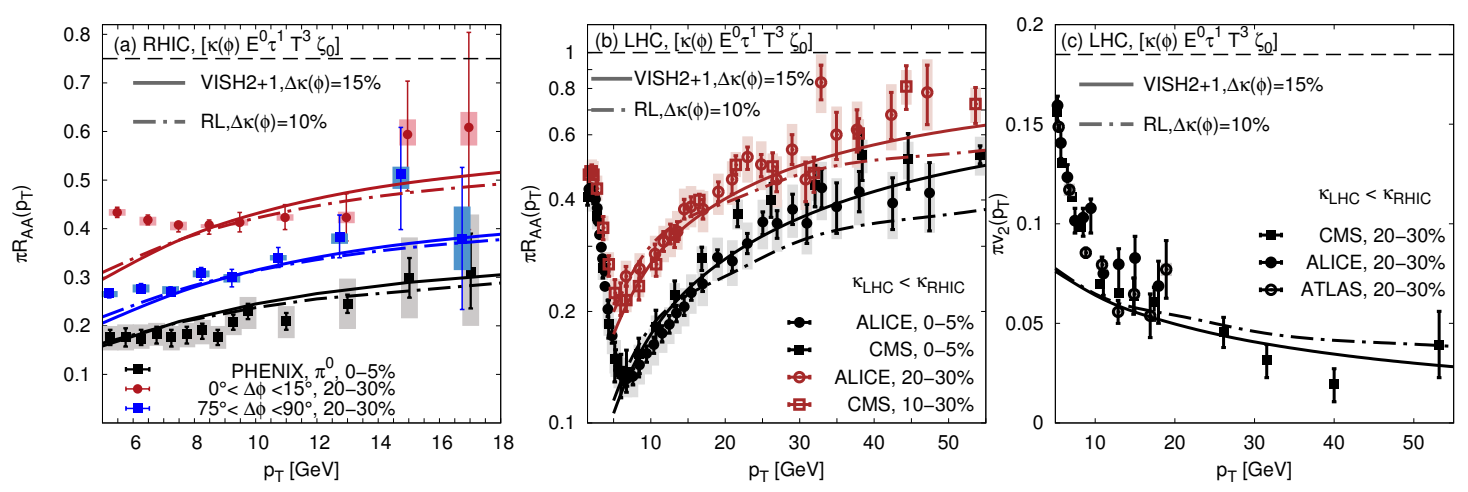

Figure 8. Azimuthal jet tomography at RHIC and LHC assuming jet-energy loss fluctuations and a moderate azimuthal dependence of the jet-medium coupling of $\Delta \kappa(\phi)=15 \%$ for the bulk QGP flow fields from VISH2 $+1[9,10]$ (solid) and $\Delta \kappa(\phi)=10 \%$ for the RL fields $[12,13]$ (dashed-dotted). Panel (a) shows the nuclear modification factor for most central collisions as well as their in-and out-of-plane contributions at RHIC, panel (b) depicts the $R_{A A}\left(p_{T}\right)$ at LHC, and panel (c) describes the high- $p_{T}$ elliptic flow at LHC energies. At LHC energies, the jet-medium coupling is reduced as compared to RHIC energies to account for the QCD running-coupling effect.

Figure 5 shows the same comparison as figure 4, however, depicting one case without jet-energy loss fluctuations (solid) and one scenario with fluctuations ( $q=1$, dashed). Clearly, the jet-energy loss fluctuations reduce the yield of the nuclear modification factors, both central and non-central, and the yield of the high- $p_{T}$ elliptic flow below $p_{T} \leq 20 \mathrm{GeV}$, while it simultaneously slightly enhances the high- $p_{T}$ elliptic flow above $p_{T}>20 \mathrm{GeV}$.

There is a certain ambiguity in the yield of both the nuclear modification factor and the high- $p_{T}$ elliptic flow that becomes obvious when comparing figure 5 (a) to figure 6 . In figure 5(a), we determine the reduction of the jet-medium coupling by assuming that the $R_{A A}\left(p_{T}=10 \mathrm{GeV}\right)=0.155$, while we supposed in figure 6 that $R_{A A}\left(p_{T}=10 \mathrm{GeV}\right)=0.186$. Both numbers are in line with the current error bars of the measured date from ALICE and CMS. This comparison demonstrates that a larger value for the nuclear modification factor implies a lower yield of the elliptic flow.

Neglecting the uncertainties given by the hydrodynamic expansions discussed above as we cannot easily assign them a theoretical error bar, figure 7 reveals that both the nonfluctuating and the fluctuating scenarios of QCDrad based on the VISH $2+1$ background fields account for the nuclear modification factors on $\chi^{2} /$ d.o.f. $<1.5$ level but fail to describe the high- $p_{T}$ elliptic flow data which is in line with the results of CUJET2.0 [39]. Please note that the discrepancies obtained in describing the high- $p_{T}$ elliptic flow certainly depend on the background flow considered, as shown in figure 4 where the RL Hydro scenario is much closer to the measured data.

In any case, there is a tendency that the high- $p_{T}$ elliptic flow is too small as compared to data. To overcome this "high- $p_{T} v_{2}$ problem" of pQCD-based jet-energy loss prescriptions, ref. [39] suggested that in addition to the vacuum running of the perturbative QCD coupling $\alpha_{s}(Q)$, there could well be a running w.r.t. the temperature $\alpha_{\text {eff }}(Q, T)[42]$ which could cause modest $(10-15 \%)$ variations of the path-averaged coupling in non-central collisions 

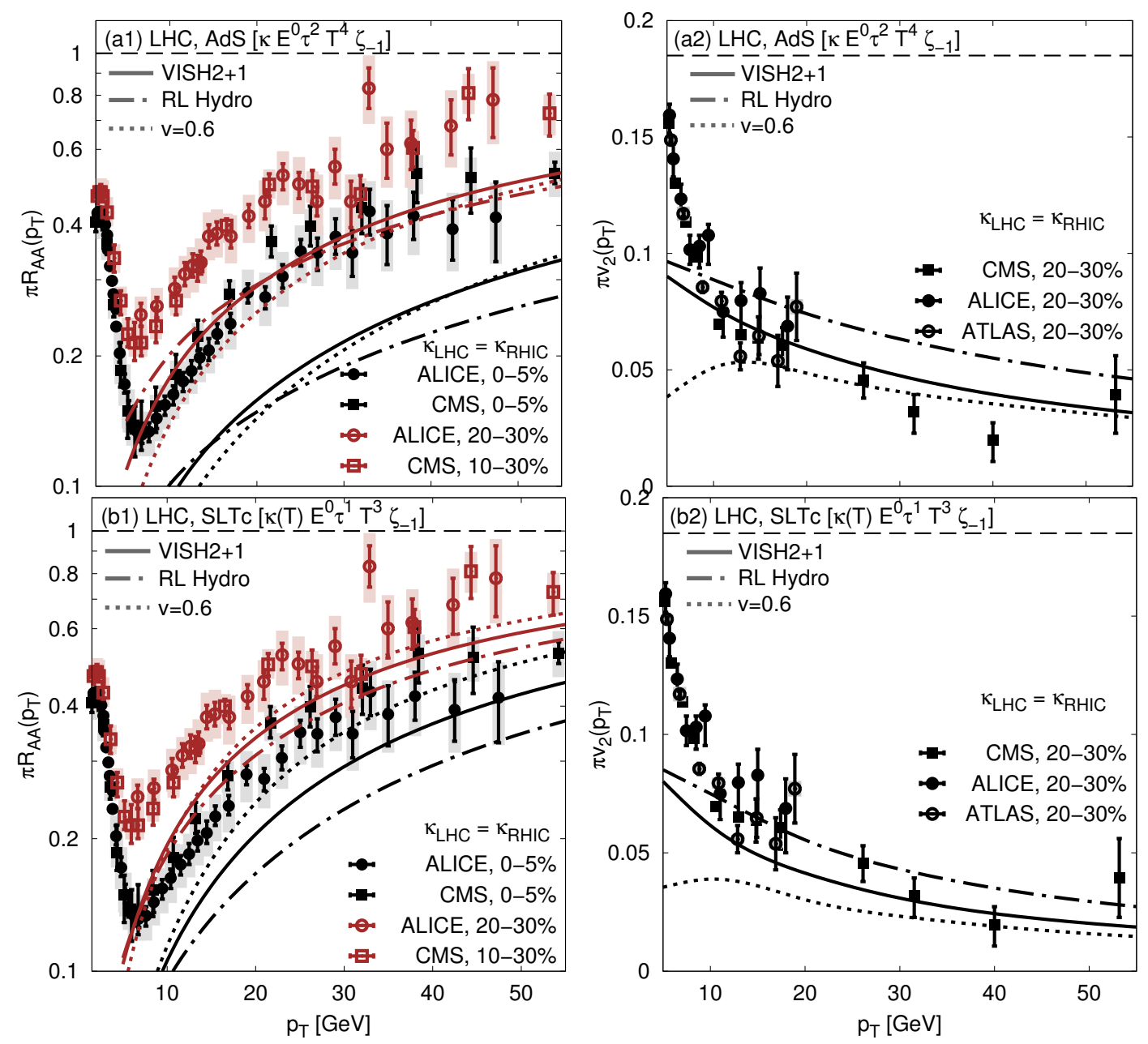

Figure 9. Azimuthal jet tomography at the LHC [17-22]. Panels (a1) and (b1) show the measured data for the pion nuclear modification factor $R_{A A}$ from ALICE [17] and CMS [19] for most central and more peripheral collisions, while panels (a2) and (b2) depict the high- $p_{T}$ elliptic flow as extracted from ALICE [18], CMS [20], and ATLAS [21, 22]. The model calculations are done to mimic a conformal AdS [45, 58-60] (upper panel) and a $T_{c}$-dominated SLTc model [23-25] (lower panel) without energy-loss fluctuations, using bulk QGP flow fields at LHC energies from viscous $\eta / s=0.08 \mathrm{VISH} 2+1[9,10]$ (solid), viscous $\eta / s=0.08 \mathrm{RL}$ Hydro $[12,13]$ (dashed-dotted), and the $v_{\perp}=0.6$ blast wave model $[14,15]$ (dotted). Here, the same jet-medium coupling $\kappa_{L H C}$ is taken at the LHC as fixed at RHIC, see text.

with a coupling constant enhanced out-of-plane. To simulate this effect, we include an azimuthal dependence of the jet-medium coupling by $\kappa(\phi)=\kappa \cdot(1+|\sin (\phi)| \cdot X)$, where $X$ is a value in percentage.

Figure 8 proves that a small azimuthal variation of $10-15 \%$ of the jet-medium coupling $\kappa$ is already sufficient to account for the high- $p_{T} v_{2}$ problem at LHC energies while simultaneously describing the nuclear modification factor in- and out-of-plane at RHIC energies as well as the nuclear modification factors at LHC energies, in line with ref. [39]. However, figure 8 also demonstrates the impact of the bulk QGP background fields. While the results 

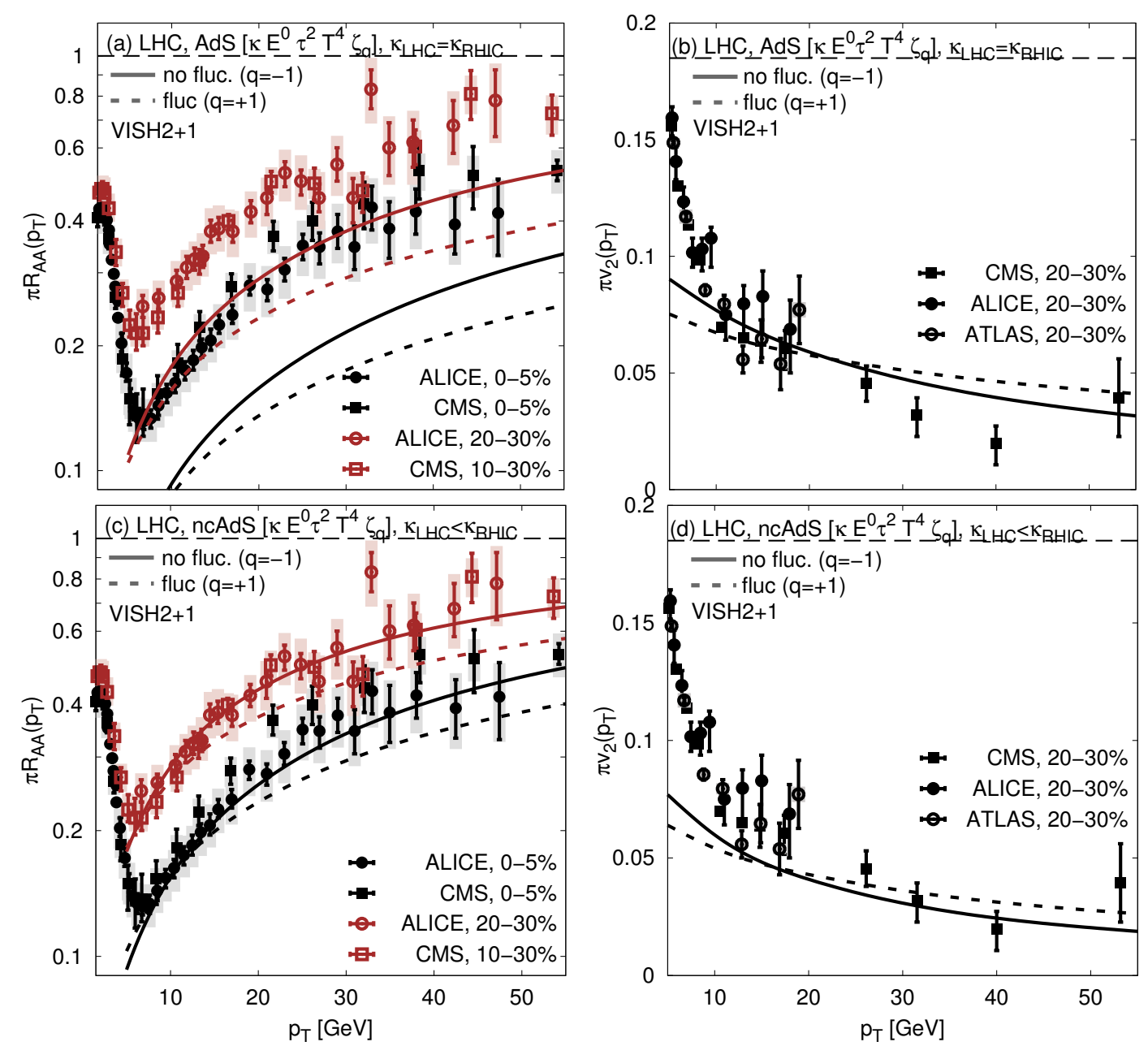

Figure 10. Azimuthal jet tomography at the LHC [17-22]. Panels (a) and (c) show the measured data for the pion nuclear modification factor $R_{A A}$ from ALICE [17] and CMS [19] for most central and more peripheral collisions, while panels (b) and (d) depict the high- $p_{T}$ elliptic flow as extracted from ALICE [18], CMS [20], and ATLAS [21, 22]. The model calculations are done for the $d E / d x=\kappa E^{0} \tau^{2} T^{4} \zeta_{q}$ scenario, comparing a fluctuating energy loss $\left(\zeta_{+1}\right.$, dashed $)$ to the nonfluctuating scenario $\left(\zeta_{-1}\right.$, solid) using bulk QGP flow fields at LHC energies from viscous $\eta / s=0.08$ VISH2 $+1[9,10]$. In the upper panel, the same $\kappa$ is taken as at RHIC, while in the lower panel, a reduced jet-medium coupling is assumed.

for VISH2 $+1[7,8,11]$ and the RL Hydro $[12,13]$ are very similar at RHIC energies, the RL Hydro background leads to nuclear modification factors that only touch the lower bounds of the measured error bars. Please note that we here assume $R_{A A}\left(p_{T}=10 \mathrm{GeV}\right)=0.155$ and jet-energy loss fluctuations. Thus, a combined jet-energy loss and bulk QGP background evolution has a much larger discriminating power than the two separate prescriptions.

In contrast to the consistent prescription of both RHIC and LHC data for the QCDrad scenario considering either no jet-energy loss fluctuations (see figure 4) or jet-energy loss fluctuations with an additional moderate azimuthal dependence of the jet-medium coupling, conformal AdS-inspired models [45-47] based on a $d E / d x \equiv \kappa x^{2} T^{4}$ and the SLTc model 


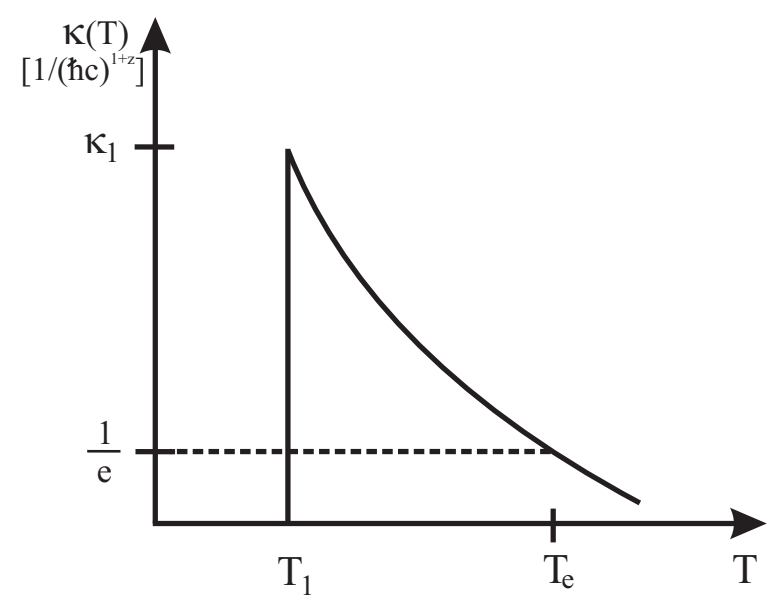

Figure 11. Sketch of an exponential temperature-dependent jet-medium coupling $\kappa(T)$, in units of $1 /(\hbar c)^{(1+z)}$, as given by eq. (3.1) assuming that the coupling is zero below a temperature $T_{1}$, peaks at $T_{1}$ with a value of $\kappa_{1}$, and falls off to a value of $1 / e$ at a temperature $T_{e}$.
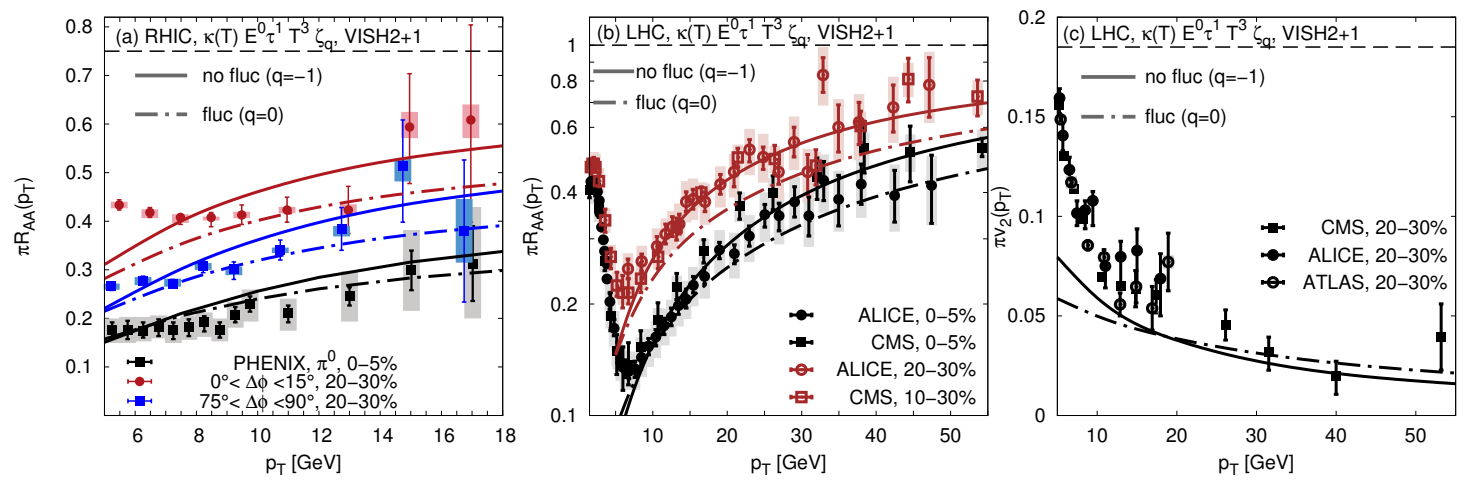

Figure 12. Azimuthal jet tomography at RHIC and LHC assuming a pQCD-like jet-energy loss and a jet-medium coupling $\kappa(T)$ showing an exponential temperature-dependence as given in eq. (3.1) with $(q=0)$ and without $(q=-1)$ additional jet-energy loss fluctuations considering the bulk QGP flow fields from VISH2+1 $[9,10]$. Panel (a) shows the nuclear modification factor for most central collisions as well as their in-and out-of-plane contributions at RHIC, panel (b) depicts the $R_{A A}\left(p_{T}\right)$ at LHC, and panel (c) describes the high- $p_{T}$ elliptic flow at LHC energies.

with an enhanced jet-medium coupling around $T_{c} \sim 170 \mathrm{GeV}$ fail the extrapolation to LHC energies for the same backgrounds considered, as shown in figure 9.

The reason is that the same jet-medium coupling $\kappa$ is assumed for RHIC and LHC in both cases, however for two different reasons: in AdS/CFT, $\kappa \propto \sqrt{\lambda}$, where $\lambda=4 \pi \alpha_{s} N_{c}$ is the 'tHooft coupling that must be $\lambda \gg 1$ to ensure applicability of classical gravity holography. For conformal AdS/CFT symmetry, $\lambda$ cannot run. In that case, as shown in figure 9(a), the AdS prescription over-quenches at the LHC (which is the well-known "surprising transparency" [29-31]) and leads to a simultaneous enhancement of the high- $p_{T}$ elliptic flow. This over-quenching behaviour was shown even for $\lambda$ as low as 1 (in static backgrounds) and quadratic curvature corrections [57] for AdS falling string models [58- 

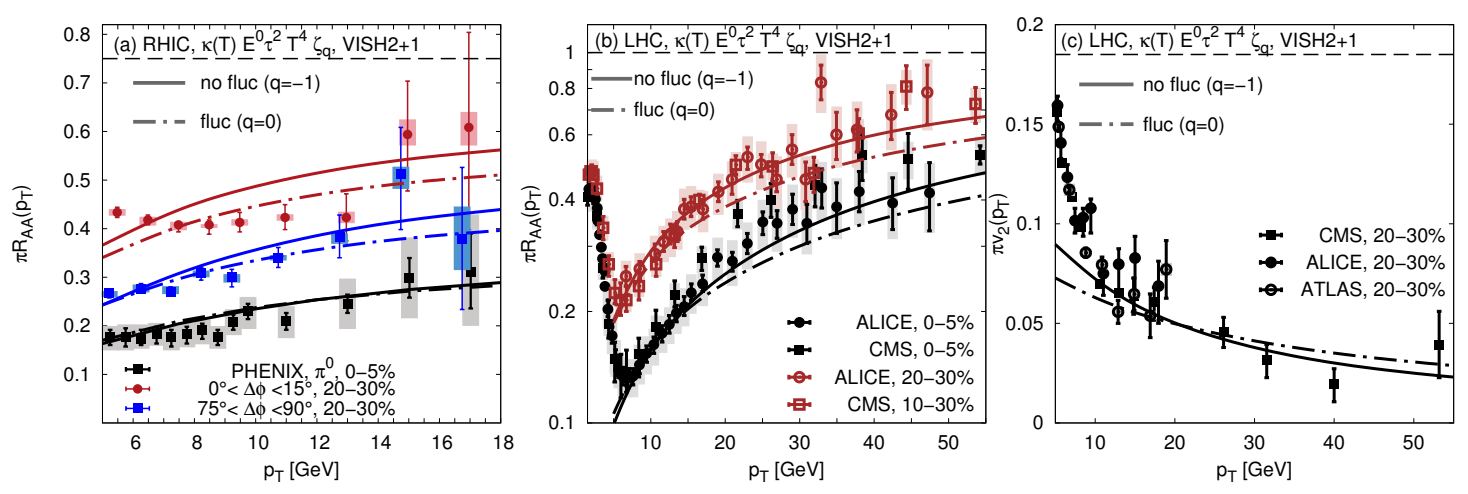

Figure 13. Azimuthal jet tomography at RHIC and LHC assuming an AdS-inspired jet-energy loss scenario with a squared path-length dependence and a jet-medium coupling $\kappa(T)$ showing an exponential temperature-dependence as given in eq. (3.1) with $(q=0)$ and without $(q=-1)$ additional jet-energy loss fluctuations considering the bulk QGP flow fields from VISH2+1 $[9,10]$. Panel (a) shows the nuclear modification factor for most central collisions as well as their in-and out-of-plane contributions at RHIC, panel (b) depicts the $R_{A A}\left(p_{T}\right)$ at LHC, and panel (c) describes the high- $p_{T}$ elliptic flow at LHC energies.

60]. Thus, jet-energy loss prescriptions based on conformal AdS/CFT are ruled out by the rapid rise of the nuclear modification factor at LHC energies.

The SLTc model [23-25], on the other hand, assumes the dominance of jet-energy loss in regions of the QGP with $T \sim T_{c} \approx 170 \mathrm{MeV}$, associating the QCD conformal anomaly near $T_{c}$ with a color magnetic monopole condensation. Scattering of color electric charged jets by color magnetic monopoles could lead to an enhancement of the jet-energy loss in the QCD crossover transition regions that have a higher spatial elliptic eccentricity than the average. Following a suggestion of ref. [23-25], we simulate this effect by a simple step function of the local jet-medium coupling with $\kappa_{c}=\kappa_{c}(113<T<173 \mathrm{MeV})=3 \kappa_{Q}$ and $\kappa_{Q}=\kappa_{Q}(T \geq$ $173 \mathrm{MeV}$ ). Assuming $\kappa_{c} / \kappa_{Q}=3$ and a transverse expanding medium, we also observe an over-quenching of the nuclear modification factors as shown in figure $9(\mathrm{~b})$. Please note that a generalization of this model with an additional collision energy $\sqrt{s}$-dependence weakening the jet-medium coupling at higher $\sqrt{s}$, and thus considering the running-coupling effect [26$33,39]$ with a $\kappa(T, \sqrt{s})$, will certainly provide an adequate prescription for the $R_{A A}$. To account for the high- $p_{T}$ elliptic flow data as well, an additional azimuthal dependence of the jet-medium coupling, as discussed in figure 8, might be necessary.

Even though present jet-energy loss models based on AdS/CFT do not consider fluctuations in the energy loss, we also examine the impact of such additional jet-energy loss fluctuations for the AdS scenario in figure 10(a) and (b). Again (cf. Figure 5), additional jet-energy loss fluctuations cause a stronger jet quenching and a flattening of the high- $p_{T}$ elliptic flow.

However, broadening the applicability of holographic models to heavy-ion collisions by allowing for non-conformal AdS/CFT prescriptions that enable a running-coupling effect with a reduced jet-medium coupling at LHC energies outweigh the over-quenching and result in a simultaneous prescription of the nuclear modification factors at different 
centralities as well as the high- $p_{T}$ elliptic flow with and without additional jet-energy loss fluctuations as shown in figure 10(c) and (d). Certainly, such an ansatz requires further generalization of the present holographic jet-quenching models to include possibly more general string initial conditions and non-conformal geometric deformations [61-63].

Despite the result of figure 9 showing that the original SLTc model with a jet-medium coupling which is non-negligible for large temperatures and enhanced in a transition area $\kappa_{c}(113<T<173 \mathrm{MeV})$ is ruled out by the measured data at the LHC, the fact reported by CUJET2.0 [39] and shown in figure 8 that a moderate azimuthal dependence of the jet-medium coupling $\kappa$ with a coupling enhanced out-of-plane can result in a simultaneous prescription of the nuclear modification factor and the high- $p_{T}$ elliptic flow at RHIC and LHC supports a jet-medium coupling enhanced for lower temperatures. The reason is that a jet traversing out-of-plane will propagate longer through a comparably cooler medium.

As the original SLTc model does not reproduce the opacity of the LHC medium appropriately, we consider below an exponentially falling ansatz for the jet-medium coupling,

$$
\kappa(T)=\kappa_{1} e^{-b\left(T-T_{1}\right)} .
$$

Here, the coupling is assumed to be zero below a certain temperature $T_{1}$, representing the freeze-out, where the coupling peaks at a value of $\kappa_{1}$ and falls off for larger temperatures to a value of $1 / e$ at a temperature $T_{e}$, see figure 11 .

Figures 12 and 13 depict the results for the nuclear modification factor and the high- $p_{T}$ elliptic flow both at RHIC and at LHC energies with and without additional jet-energy loss fluctuations, considering the bulk QGP flow fields from VISH2+1 $[9,10]$ for either a pQCD-based jet-energy loss scenario, figure 12, or an AdS-inspired prescription with a squared path-length dependence, figure 13.

Please note that in contrast to previous figures there is only one fixing point considered here, $R_{A A}\left(p_{T}=7.5 \mathrm{GeV}, \mathrm{RHIC}\right)=0.2$. Applying the exponentially falling jet-medium coupling $\kappa(T)$, a reduction of the effective jet-medium coupling at the LHC is intrinsic as an LHC-jet propagates longer through a high-temperature region with a smaller coupling. An additional reduction of the jet-medium coupling is not needed. Of course, the SLTc model also shows such an intrinsic weakening of the jet-medium coupling at the LHC. However, as proven in figure 9, this intrinsic reduction is not sufficient to account for the transparency at LHC energies [26-33, 39].

Surprisingly enough, the results shown in figures 12 and 13 with a $T_{1}=160 \mathrm{MeV}$ for all scenarios point to having a comparatively low value for $T_{e}$, see table 1 , indicating that the high-temperature medium is basically transparent [26-33, 39].

Comparing figures 12 and 13, both the pQCD-based and the AdS-inspired models describe the measured data within the present error bars. This reinforces our conclusion that the path-length exponent cannot yet be constrained to a range narrower than $z=$ $[0-2]$. Please note that $z=0$ seems to be disfavored as shown by the QCDel results but cannot definitely be excluded.

Please also note that the exponential falling $\kappa(T)$ as given by eq. (3.1) is one possible ansatz for a jet-medium coupling describing the transparency of the LHC medium (via the 


\begin{tabular}{|c|r|c|c|}
\hline scenario & $q$ & $T_{e}[\mathrm{MeV}]$ & $\kappa_{1}\left(T_{1}\right)$ \\
\hline pQCD-like & -1 & 250 & 1.281 \\
\hline pQCD-like & 0 & 270 & 2.134 \\
\hline AdS-inspired & -1 & 220 & 0.589 \\
\hline AdS-inspired & 0 & 230 & 0.956 \\
\hline
\end{tabular}

Table 1. The parameters for the exponential temperature-dependent jet-medium coupling $\kappa(T)$ as given by eq. (3.1) and shown in figures 12 and 13 with $T_{1}=160 \mathrm{MeV}$ and $\kappa_{e}\left(T_{e}\right)=1 / e=0.37$ [in units of $\left.1 /(\hbar c)^{(1+z)}\right]$.

$\left.R_{A A}\left(p_{T}\right)\right)$ and the high- $p_{T}$ elliptic flow appropriately. However, other ansaetze might work as well.

Finally we note that at both RHIC and LHC energies, the magnitude of the nuclear modification factors in the intermediate ("IM") $2<p_{T}<7 \mathrm{GeV}$ kinematic region is under-predicted by all jet-quenching models considered here. This "IM" region interpolates between the perfect fluid low- $p_{T}<2 \mathrm{GeV}$ infrared ("IR") range and the high- $p_{T}>7 \mathrm{GeV}$ ultraviolet ("UV") perturbative QCD jet-quenching range. A proper theory of jet quenching in the non-equilibrium QGP "IM" range remains a formidable challenge.

\section{Conclusions}

We compare recent data on the nuclear modification factor and the high- $p_{T}$ elliptic flow measured at RHIC [16] and LHC energies [17-22] to a broad class of jet-energy independent energy-loss models (see table 2) with $d E / d x=\kappa(T) E^{a=0} x^{z} T^{c} \zeta_{q}$, labelled by $(z, c, q)$, including jet-energy loss fluctuations for $q>-1$. In particular, we study (a) a linear, radiative pQCD-like jet-energy loss with running coupling [26-28, 32, 33, 39], (b) a linear, elastic pQCD-like jet-energy loss with running coupling [29-31], (c) an AdS/CFT-inspired, quadratic jet-energy loss, (d) a $T_{c}$-dominated energy-loss model (SLTc) [23-25], (e) an energy-loss prescription based on a moderate azimuthal dependence of the jet-medium coupling [39], and (f) an energy-loss scenario with a temperature-dependent jet-medium coupling $\kappa(T)$ dropping exponentially for large temperatures. All those models are combined with several recent transverse and Bjorken expanding, collective flow backgrounds [7-15].

We find (see table 3 ) that (1) running coupling energy-loss models with $(0,1,3)$ motivated by perturbative QCD appear to be favored, (2) conformal AdS/CFT-inspired jetenergy loss scenarios are ruled out by the reduction of coupling required to fit LHC data, and $(3)$ a realistic $[(2+1)$ d] QGP flow background is essential to account for the dependence of the data on transverse momentum $p_{T}$, azimuth $\phi$, impact parameter $b$, and collision energy $\sqrt{s}$.

We further explore different possible deformations of the models that could reduce the discrepancies with the combined RHIC and LHC data. The simplest solution utilizes either (viscous) VISH2+1 [7-10] or RL $[12,13]$ hydrodynamic fields and corresponds to a radiative pQCD-like energy loss with $d E / d x=\kappa x^{1} T^{3} \zeta_{-1},(1,3,-1)$, with $\zeta_{-1}=1$ neglecting jet- 
energy loss fluctuations. While this fits, it is theoretically not compelling. A second solution includes those energy-loss fluctuations $(q=0,1)$ for $(1,3, q)$ pQCD-like modelling, but allows for $\sim 10-15 \%$ variation of the jet-medium coupling along different paths relative to the reaction plane. The exact value of the variation depends on the QGP flow fields used $[7-10,12,13]$. This class of solutions is similar to the one recently proposed by the more detailed CUJET2.0 model [39]. It remains to be seen whether the combined temperature and scale running of jet-medium coupling $\alpha_{\text {eff }}(Q, T)[39,42]$ can be more rigorously justified.

A third class of $(1,3, q)$ solutions assumes a more radical temperature-dependent jetmedium coupling $\kappa(T)$ with not only an enhancement of the coupling near $T_{c}$, in the spirit of the SLTc scenario, but requiring an exponential suppression of $\kappa(T)$ at high temperatures that is rather puzzling from both a $\mathrm{pQCD}$ and an AdS points of view. A fourth class of solutions also assumes a temperature-dependent jet-medium coupling $\kappa(T)$ with an enhancement of the coupling near $T_{c}$ as given by the SLTc scenario, but requiring an additional reduction of the magnitude of $\kappa(T)$ at the LHC.

Finally, a fifth class of AdS-inspired solutions with a quadratic jet-path length dependence $d E / d x=\kappa(T) x^{2} T^{4} \zeta_{q}$ premises a strong non-conformal reduction of jet-medium coupling by a factor of two at LHC energies. Thus far, no holographic model has predicted such strong conformal breaking effects.

Please see table 2 for a summary of the relative success and failure of the different models surveyed in table 3 .

Given the current landscape of jet-medium modelling in table 3 and the uncertainties in justifying deformations of current models required to fit the data, especially the LHC high- $p_{T}$ elliptic moment $v_{2}\left(p_{T}\right)$, we cannot constrain the path-length exponent $z$ of the jet-energy loss to a range narrower than $z=[0-2]$.

\section{Acknowledgments}

We are especially grateful to P. Romatschke, U. Heinz, and C. Shen for making their hydrodynamic field grids available. Discussions with J. Xu, A. Ficnar, A. Buzzatti, W. Horowitz, J. Liao, D. Molnar, and X.-N. Wang in the JET Collaboration have been particularly valuable. BB acknowledges financial support received from the Helmholtz International Centre for FAIR within the framework of the LOEWE program (Landesoffensive zur Entwicklung Wissenschaftlich-Ökonomischer Exzellenz) launched by the State of Hesse. MG acknowledges support from the US-DOE Nuclear Science Grant No. DE-FG02-93ER40764 and No. DE-AC02-05CH11231 within the framework of the JET Topical Collaboration [6]. The authors also thank the Yukawa Institute for Theoretical Physics, Kyoto University, where part of this work was completed during the YITP-T-13-05 on "New Frontiers in QCD". MG is grateful for partial support from the MTA Wigner RCP, Budapest, during the second half of his sabbatical leave in 2014, where this work was finalized. 


\section{A Jet+bulk models used in the present survey}

\begin{tabular}{|c|c|c|c|c|c|c|c|}
\hline$\#$ & name & fluct. & $(z, c, q)$ & temp. profile & $\kappa_{\mathrm{RHIC}}$ & $\kappa_{\mathrm{LHC}}$ & Fig. \# \\
\hline 1 & QCDrad & no & $(1,3,-1)$ & VISH $2+1$ & 0.380 & 0.167 & $1,4,5$ \\
\hline $1 \mathrm{a}$ & QCDrad & no & $(1,3,-1)$ & VISH2+1 & 0.380 & 0.136 & 1,6 \\
\hline 2 & QCDrad & no & $(1,3,-1)$ & RL Hydro & 0.477 & 0.241 & 1,4 \\
\hline 3 & QCDrad & no & $(1,3,-1)$ & $v=0.6$ & 3.182 & 2.096 & 1,4 \\
\hline 4 & QCDel & no & $(0,2,-1)$ & VISH2+1 & 0.887 & 0.483 & 1,4 \\
\hline 5 & QCDel & no & $(0,2,-1)$ & RL Hydro & 1.497 & 0.906 & 1,4 \\
\hline 6 & QCDel & no & $(0,2,-1)$ & $v=0.6$ & 5.713 & 5.024 & 1,4 \\
\hline 7 & AdS & no & $(2,4,-1)$ & VISH2+1 & 0.092 & 0.092 & 1,9 \\
\hline 8 & AdS & no & $(2,4,-1)$ & RL Hydro & 0.145 & 0.145 & 1,9 \\
\hline 9 & AdS & no & $(2,4,-1)$ & $v=0.6$ & 1.911 & 1.911 & 1,9 \\
\hline 10 & SLTc & no & $(1,3,-1)$ & VISH2+1 & 0.167 & 0.167 & 1,9 \\
\hline 11 & SLTc & no & $(1,3,-1)$ & RL Hydro & 0.330 & 0.330 & 1,9 \\
\hline 12 & SLTc & no & $(1,3,-1)$ & $v=0.6$ & 1.591 & 1.591 & 1,9 \\
\hline 13 & QCDrad & yes & $(1,3,+1)$ & VISH2+1 & 0.718 & 0.349 & 2,5 \\
\hline $13 \mathrm{a}$ & QCDrad & yes & $(1,3,+1)$ & VISH2+1 & 0.718 & 0.269 & 2,6 \\
\hline 14 & QCDel & yes & $(1,3,+1)$ & VISH2+1 & 1.615 & 1.024 & 2,5 \\
\hline 15 & AdS & yes & $(2,4,+1)$ & VISH2+1 & 0.283 & 0.283 & $2,10(a, b)$ \\
\hline 16 & ncAdS & no & $(2,4,-1)$ & VISH2+1 & 0.092 & 0.047 & $2,10(\mathrm{c}, \mathrm{d})$ \\
\hline 17 & ncAdS & yes & $(2,4,+1)$ & VISH2+1 & 0.283 & 0.111 & $2,10(\mathrm{c}, \mathrm{d})$ \\
\hline 18 & $\kappa(\phi)$ QCDrad & yes & $(1,3,0)$ & VISH2+1 & 0.543 & 0.235 & 8 \\
\hline 19 & $\kappa(\phi)$ QCDrad & yes & $(1,3,0)$ & RL Hydro & 0.776 & 0.345 & 8 \\
\hline 20 & exp. $\kappa(T)$ QCDrad & no & $(1,3,-1)$ & VISH $2+1$ & $\kappa_{1}=1.281$ & $\kappa_{1}=1.281$ & 12 \\
\hline 21 & $\exp . \kappa(T)$ QCDrad & yes & $(1,3,0)$ & VISH2+1 & $\kappa_{1}=2.134$ & $\kappa_{1}=2.134$ & 12 \\
\hline 22 & exp. $\kappa(T)$ ncAdS & no & $(2,4,-1)$ & VISH2+1 & $\kappa_{1}=0.589$ & $\kappa_{1}=0.589$ & 13 \\
\hline 23 & exp. $\kappa(T)$ ncAdS & yes & $(2,4,0)$ & VISH2+1 & $\kappa_{1}=0.956$ & $\kappa_{1}=0.956$ & 13 \\
\hline
\end{tabular}

Table 2. Parameters of the jet-energy loss models and bulk-temperature fields considered in the present survey. The columns show the model identifier name, whether jet-energy loss fluctuations are considered, the path-length exponent $z$, the temperature exponent $c$, and the energy-loss fluctuation parameter $q$ as well as the bulk-temperature $T(\vec{x}, t)$ field assumed. The effective jet-medium coupling at RHIC obtained by a single fit to central $R_{A A}\left(p_{T}=7.5 \mathrm{GeV}\right)$ data are listed in column 6 . Column 7 shows the values of the LHC jet-medium coupling used in the figures listed in column 8 . See text for details. 


\begin{tabular}{|c|c|c|c|c|c|c|c|}
\hline $\begin{array}{c}\text { Scenario } \\
\#\end{array}$ & \multicolumn{3}{|c|}{ RHIC } & \multicolumn{3}{|c|}{ LHC } & Score \\
\hline 1 & $R_{\text {AA }}^{\text {centr }}$ & $R_{\text {AA }}^{\text {in,periph }}$ & $R_{\text {AAt,periph }}^{\text {out }}$ & $R_{A A}^{\text {centr }}$ & $R_{A A}^{\text {periph }}$ & $v_{2}^{\text {periph }}$ & Sum \\
\hline 1 a & $\checkmark$ & $\checkmark$ & $\checkmark$ & $\checkmark$ & $\checkmark$ & $(\checkmark)$ & 5 \\
\hline 2 & $\checkmark$ & $\checkmark$ & $\checkmark$ & $(\checkmark)$ & $(\checkmark)$ & $(\checkmark)$ & 3 \\
\hline 3 & $(\checkmark)$ & $\checkmark$ & no & $\checkmark$ & $\checkmark$ & no & 1 \\
\hline 4 & $\checkmark$ & $\checkmark$ & $\checkmark$ & $(\checkmark)$ & $(\checkmark)$ & $(\checkmark)$ & 3 \\
\hline 5 & $\checkmark$ & $\checkmark$ & $\checkmark$ & $\checkmark$ & $(\checkmark)$ & $(\checkmark)$ & 4 \\
\hline 6 & $\checkmark$ & no & $\checkmark$ & $(\checkmark)$ & $(\checkmark)$ & no & 0 \\
\hline 7 & $\checkmark$ & $\checkmark$ & $\checkmark$ & no & no & $\checkmark$ & 2 \\
\hline 8 & $\checkmark$ & $\checkmark$ & $\checkmark$ & no & no & no & 0 \\
\hline 9 & $\checkmark$ & $\checkmark$ & no & no & no & $(\checkmark)$ & -1 \\
\hline 10 & $\checkmark$ & $\checkmark$ & $\checkmark$ & no & no & $\checkmark$ & 2 \\
\hline 11 & $\checkmark$ & $\checkmark$ & $\checkmark$ & no & no & $\checkmark$ & 2 \\
\hline 12 & $(\checkmark)$ & no & no & no & no & no & -5 \\
\hline 13 & $\checkmark$ & $(\checkmark)$ & $(\checkmark)$ & $(\checkmark)$ & no & $(\checkmark)$ & 0 \\
\hline $13 \mathrm{a}$ & $\checkmark$ & $(\checkmark)$ & $(\checkmark)$ & $\checkmark$ & $(\checkmark)$ & $(\checkmark)$ & 2 \\
\hline 14 & $\checkmark$ & no & no & $\checkmark$ & no & no & -2 \\
\hline 15 & $\checkmark$ & $\checkmark$ & $(\checkmark)$ & no & no & $(\checkmark)$ & 0 \\
\hline 16 & $\checkmark$ & $(\checkmark)$ & $\checkmark$ & $\checkmark$ & $\checkmark$ & $\checkmark$ & 5 \\
\hline 17 & $\checkmark$ & $\checkmark$ & $(\checkmark)$ & no & no & $\checkmark$ & 1 \\
\hline 18 & $\checkmark$ & $\checkmark$ & $\checkmark$ & $\checkmark$ & $\checkmark$ & $\checkmark$ & 6 \\
\hline 19 & $\checkmark$ & $\checkmark$ & $\checkmark$ & no & no & $(\checkmark)$ & 1 \\
\hline 20 & $\checkmark$ & $(\checkmark)$ & $\checkmark$ & $\checkmark$ & $\checkmark$ & $\checkmark$ & 5 \\
\hline 21 & $\checkmark$ & $\checkmark$ & $(\checkmark)$ & $(\checkmark)$ & no & $\checkmark$ & 1 \\
\hline 22 & $\checkmark$ & $\checkmark$ & $(\checkmark)$ & $\checkmark$ & $\checkmark$ & $\checkmark$ & 5 \\
\hline 23 & $\checkmark$ & $\checkmark$ & $\checkmark$ & $(\checkmark)$ & no & $\checkmark$ & 3 \\
\hline
\end{tabular}

Table 3. Relative matrix of success and failure of the jet+bulk models surveyed in table 2 based on comparisons of the results to RHIC and LHC data for $R_{A A}\left(p_{T}, \phi, b, \sqrt{s}\right)$ and high- $p_{T} v_{2}\left(p_{T}, \phi, b, \sqrt{s}\right)$. The last column shows a score given by Score = number of checks - number of no's. A "(check)" indicates an inconclusive judgement of success and is given a zero weight. Model 18 has highest score 6 , while the models $1,3,16,20$, and 22 are tied at score 5 . See text for discussion. 
Open Access. This article is distributed under the terms of the Creative Commons Attribution License (CC-BY 4.0), which permits any use, distribution and reproduction in any medium, provided the original author(s) and source are credited.

\section{References}

[1] M. Gyulassy, I. Vitev, X.-N. Wang and B.-W. Zhang, Jet quenching and radiative energy loss in dense nuclear matter, in Quark gluon plasma, R.C. Hwa et al. eds., pg. 123, nucl-th/0302077 [INSPIRE].

[2] M. Gyulassy, Theory of high-energy A+A at RHIC., Lect. Notes Phys. 583 (2002) 37 [nucl-th/0106072] [INSPIRE].

[3] I. Vitev and M. Gyulassy, High $p_{T}$ tomography of $d+A u$ and Au+Au at SPS, RHIC and LHC, Phys. Rev. Lett. 89 (2002) 252301 [hep-ph/0209161] [INSPIRE].

[4] M. Gyulassy, P. Levai and I. Vitev, Reaction operator approach to nonAbelian energy loss, Nucl. Phys. B 594 (2001) 371 [nucl-th/0006010] [INSPIRE].

[5] X.-N. Wang and M. Gyulassy, Gluon shadowing and jet quenching in $A+A$ collisions at $\sqrt{s}=200 \mathrm{GeV}$, Phys. Rev. Lett. 68 (1992) 1480 [INSPIRE].

[6] (JET) Topical Collaboration on Jet and Electromagnetic Tomography, http://jet.lbl.gov/main.

[7] H. Song and U.W. Heinz, Causal viscous hydrodynamics in $2+1$ dimensions for relativistic heavy-ion collisions, Phys. Rev. C $7 \mathbf{7}$ (2008) 064901 [arXiv:0712.3715] [INSPIRE].

[8] H. Song and U.W. Heinz, Multiplicity scaling in ideal and viscous hydrodynamics, Phys. Rev. C 78 (2008) 024902 [arXiv:0805.1756] [INSPIRE].

[9] C. Shen, U. Heinz, P. Huovinen and H. Song, Radial and elliptic flow in Pb+Pb collisions at the Large Hadron Collider from viscous hydrodynamic, Phys. Rev. C 84 (2011) 044903 [arXiv: 1105.3226] [INSPIRE].

[10] Z. Qiu, C. Shen and U. Heinz, Hydrodynamic elliptic and triangular flow in $\mathrm{Pb}-\mathrm{Pb}$ collisions at $\sqrt{s}=2.76 \mathrm{~A} \mathrm{TeV}$, Phys. Lett. B 707 (2012) 151 [arXiv:1110.3033] [INSPIRE].

[11] C. Shen, U. Heinz, P. Huovinen and H. Song, Systematic parameter study of hadron spectra and elliptic flow from viscous hydrodynamic simulations of Au+Au collisions at $\sqrt{s_{N N}}=200 \mathrm{GeV}$, Phys. Rev. C 82 (2010) 054904 [arXiv:1010.1856] [INSPIRE].

[12] M. Luzum and P. Romatschke, Conformal Relativistic Viscous Hydrodynamics: Applications to RHIC results at $\sqrt{s_{N N}}=200 \mathrm{GeV}$, Phys. Rev. C 78 (2008) 034915 [Erratum ibid. C 79 (2009) 039903] [arXiv:0804.4015] [INSPIRE].

[13] M. Luzum and P. Romatschke, Viscous Hydrodynamic Predictions for Nuclear Collisions at the LHC, Phys. Rev. Lett. 103 (2009) 262302 [arXiv:0901.4588] [inSPIRE].

[14] M. Gyulassy, I. Vitev, X.-N. Wang and P. Huovinen, Transverse expansion and high $p_{T}$ azimuthal asymmetry at RHIC, Phys. Lett. B 526 (2002) 301 [nucl-th/0109063] [INSPIRE].

[15] M. Gyulassy, I. Vitev and X.N. Wang, High $p_{T}$ azimuthal asymmetry in noncentral $A+A$ at RHIC, Phys. Rev. Lett. 86 (2001) 2537 [nucl-th/0012092] [INSPIRE].

[16] PHENIX collaboration, A. Adare et al., Neutral pion production with respect to centrality and reaction plane in Au+Au collisions at $\sqrt{s_{N N}}=200 \mathrm{GeV}$, Phys. Rev. C 87 (2013) 034911 [arXiv: 1208.2254] [INSPIRE]. 
[17] ALICE collaboration, Centrality Dependence of Charged Particle Production at Large Transverse Momentum in Pb-Pb Collisions at $\sqrt{s_{\mathrm{NN}}}=2.76 \mathrm{TeV}$, Phys. Lett. B 720 (2013) 52 [arXiv: 1208.2711] [INSPIRE].

[18] ALICE collaboration, Anisotropic flow of charged hadrons, pions and (anti-)protons measured at high transverse momentum in Pb-Pb collisions at $\sqrt{s_{N N}}=2.76 \mathrm{TeV}$, Phys. Lett. B 719 (2013) 18 [arXiv:1205.5761] [INSPIRE].

[19] CMS collaboration, Study of high-pT charged particle suppression in $\mathrm{PbPb}$ compared to $p p$ collisions at $\sqrt{s_{N N}}=2.76$ TeV, Eur. Phys. J. C 72 (2012) 1945 [arXiv:1202.2554] [INSPIRE].

[20] CMS collaboration, Measurement of the azimuthal anisotropy of neutral pions in $\mathrm{PbPb}$ collisions at $\sqrt{s_{N N}}=2.76 \mathrm{TeV}$, Phys. Rev. Lett. 110 (2013) 042301 [arXiv:1208.2470] [INSPIRE].

[21] ATLAS collaboration, Measurement of the azimuthal anisotropy for charged particle production in $\sqrt{s_{N N}}=2.76 \mathrm{TeV}$ lead-lead collisions with the ATLAS detector, Phys. Rev. C 86 (2012) 014907 [arXiv: 1203.3087] [INSPIRE].

[22] ATLAS collaboration, Measurement of the pseudorapidity and transverse momentum dependence of the elliptic flow of charged particles in lead-lead collisions at $\sqrt{s_{N N}}=2.76 \mathrm{TeV}$ with the ATLAS detector, Phys. Lett. B 707 (2012) 330 [arXiv:1108.6018] [INSPIRE].

[23] J. Liao and E. Shuryak, Angular Dependence of Jet Quenching Indicates Its Strong Enhancement Near the QCD Phase Transition, Phys. Rev. Lett. 102 (2009) 202302 [arXiv: 0810.4116] [INSPIRE].

[24] X. Zhang and J. Liao, Hard Probe of Geometry and Fluctuations from RHIC to LHC, Phys. Rev. C 89 (2014) 014907 [arXiv: 1208.6361] [INSPIRE].

[25] X. Zhang and J. Liao, Event-by-event azimuthal anisotropy of jet quenching in relativistic heavy ion collisions, Phys. Rev. C 87, 044910 (2013) 044910 [arXiv:1210.1245] [INSPIRE].

[26] B. Betz and M. Gyulassy, Azimuthal Jet Tomography of Quark Gluon Plasmas at RHIC and $L H C$, arXiv: 1305.6458 [INSPIRE].

[27] B. Betz and M. Gyulassy, Examining a reduced jet-medium coupling in $\mathrm{Pb}+\mathrm{Pb}$ collisions at the Large Hadron Collider, Phys. Rev. C 86 (2012) 024903 [arXiv:1201.0281] [InSPIRE].

[28] B. Betz, M. Gyulassy and G. Torrieri, Fourier Harmonics of High-pT Particles Probing the Fluctuating Intitial Condition Geometries in Heavy-Ion Collisions, Phys. Rev. C 84 (2011) 024913 [arXiv: 1102 .5416] [INSPIRE].

[29] W.A. Horowitz and M. Gyulassy, The Surprising Transparency of the sQGP at LHC, Nucl. Phys. A 872 (2011) 265 [arXiv:1104.4958] [INSPIRE].

[30] W.A. Horowitz and M. Gyulassy, Quenching and Tomography from RHIC to LHC, J. Phys. G 38 (2011) 124114 [arXiv:1107.2136] [INSPIRE].

[31] W.A. Horowitz, Testing $p Q C D$ and AdS/CFT Energy Loss at RHIC and LHC, AIP Conf. Proc. 1441 (2012) 889 [arXiv:1108.5876] [INSPIRE].

[32] A. Buzzatti and M. Gyulassy, A running coupling explanation of the surprising transparency of the QGP at LHC, Nucl. Phys. A 904-905 (2013) 779c [arXiv:1210.6417] [INSPIRE].

[33] A. Buzzatti and M. Gyulassy, Jet Flavor Tomography of Quark Gluon Plasmas at RHIC and LHC, Phys. Rev. Lett. 108 (2012) 022301 [arXiv:1106.3061] [INSPIRE]. 
[34] B.G. Zakharov, Nuclear modification factor for light and heavy flavors within $p Q C D$ and recent data from the LHC, JETP Lett. 96 (2013) 616 [arXiv:1210.4148] [INSPIRE].

[35] B.G. Zakharov, Jet quenching with running coupling including radiative and collisional energy losses, JETP Lett. 88 (2008) 781 [arXiv:0811.0445] [INSPIRE].

[36] B.G. Zakharov, Nuclear suppression of light hadrons and single electrons at the RHIC and LHC, J. Phys. G 40 (2013) 085003 [arXiv:1304.5742] [InSPIRE].

[37] R.A. Lacey, N.N. Ajitanand, J.M. Alexander, J. Jia and A. Taranenko, Scaling patterns for the suppression of charged hadron yields in $\mathrm{Pb}+\mathrm{Pb}$ collisions at $\sqrt{s_{N N}}=2.76 \mathrm{TeV}$ : Constraints on transport coefficients, arXiv:1202.5537 [INSPIRE].

[38] S. Pal and M. Bleicher, Suppression of high transverse momenta hadrons in $\mathrm{Pb}+\mathrm{Pb}$ collisions at LHC, Phys. Lett. B 709 (2012) 82 [arXiv:1201.2546] [INSPIRE].

[39] J. Xu, A. Buzzatti and M. Gyulassy, Azimuthal jet flavor tomography with CUJET2.0 of nuclear collisions at RHIC and LHC, arXiv:1402.2956 [INSPIRE].

[40] D. Molnar and D. Sun, High-pT suppression and elliptic flow from radiative energy loss with realistic bulk medium expansion, arXiv:1305.1046 [INSPIRE].

[41] D. Molnar and D. Sun, Realistic medium-averaging in radiative energy loss, Nucl. Phys. A910-911 (2013) 486 [arXiv:1209.2430] [INSPIRE].

[42] O. Kaczmarek, F. Karsch, F. Zantow and P. Petreczky, Static quark anti-quark free energy and the running coupling at finite temperature, Phys. Rev. D 70 (2004) 074505 [Erratum ibid. D 72 (2005) 059903] [hep-lat/0406036] [INSPIRE].

[43] S.A. Bass et al., Systematic Comparison of Jet Energy-Loss Schemes in a realistic hydrodynamic medium, Phys. Rev. C 79 (2009) 024901 [arXiv:0808.0908] [InSPIRE].

[44] C. Nonaka and S.A. Bass, Space-time evolution of bulk QCD matter, Phys. Rev. C 75 (2007) 014902 [nucl-th/0607018] [INSPIRE].

[45] C. Marquet and T. Renk, Jet quenching in the strongly-interacting quark-gluon plasma, Phys. Lett. B 685 (2010) 270 [arXiv:0908.0880] [InSPIRE].

[46] J. Jia, W.A. Horowitz and J. Liao, A study of the correlations between jet quenching observables at RHIC, Phys. Rev. C 84 (2011) 034904 [arXiv: 1101.0290] [INSPIRE].

[47] J. Jia and R. Wei, Dissecting the role of initial collision geometry for jet quenching observables in relativistic heavy ion collisions, Phys. Rev. C 82 (2010) 024902 [arXiv: 1005.0645] [INSPIRE].

[48] T. Renk, Constraining the Physics of Jet Quenching, Phys. Rev. C 85 (2012) 044903 [arXiv: 1112.2503] [INSPIRE].

[49] T. Renk et al., Systematics of parton-medium interaction from RHIC to LHC, J. Phys. G 38 (2011) 124089 [arXiv:1106.2392] [INSPIRE].

[50] T. Renk, H. Holopainen, R. Paatelainen and K.J. Eskola, Systematics of the charged-hadron $P_{T}$ spectrum and the nuclear suppression factor in heavy-ion collisions from $\sqrt{s}=200 \mathrm{GeV}$ to $\sqrt{s}=2.76$ TeV, Phys. Rev. C 84 (2011) 014906 [arXiv:1103.5308] [inSPIRE].

[51] X.-F. Chen, T. Hirano, E. Wang, X.-N. Wang and H. Zhang, Suppression of high $p_{T}$ hadrons in Pb+Pb Collisions at LHC, Phys. Rev. C 84 (2011) 034902 [arXiv:1102 .5614] [INSPIRE].

[52] H. Niemi, K.J. Eskola and P.V. Ruuskanen, Elliptic flow in nuclear collisions at the Large Hadron Collider, Phys. Rev. C 79 (2009) 024903 [arXiv:0806.1116] [INSPIRE]. 
[53] R.J. Fries and R. Rodriguez, Event-by-Event Jet Quenching and Higher Fourier Moments of Hard Probes, Nucl. Phys. A 855 (2011) 424 [arXiv:1012.3950] [INSPIRE].

[54] R. Baier, A.H. Mueller and D. Schiff, How does transverse (hydrodynamic) flow affect jet-broadening and jet-quenching?, Phys. Lett. B 649 (2007) 147 [nucl-th/0612068] [INSPIRE].

[55] M. Djordjevic and M. Gyulassy, Heavy quark radiative energy loss in QCD matter, Nucl. Phys. A 733 (2004) 265 [nucl-th/0310076] [INSPIRE].

[56] S. Wicks, W. Horowitz, M. Djordjevic and M. Gyulassy, Elastic, inelastic and path length fluctuations in jet tomography, Nucl. Phys. A 784 (2007) 426 [nucl-th/0512076] [InSPIRE].

[57] A. Ficnar, J. Noronha and M. Gyulassy, Falling Strings and Light Quark Jet Quenching at LHC, Nucl. Phys. A 910-911 (2013) 252 [arXiv: 1208.0305] [INSPIRE].

[58] S.S. Gubser, D.R. Gulotta, S.S. Pufu and F.D. Rocha, Gluon energy loss in the gauge-string duality, JHEP 10 (2008) 052 [arXiv:0803.1470] [INSPIRE].

[59] P.M. Chesler, K. Jensen, A. Karch and L.G. Yaffe, Light quark energy loss in strongly-coupled $N=4$ supersymmetric Yang-Mills plasma, Phys. Rev. D 79 (2009) 125015 [arXiv:0810.1985] [INSPIRE].

[60] A. Ficnar, AdS/CFT Energy Loss in Time-Dependent String Configurations, Phys. Rev. D 86 (2012) 046010 [arXiv:1201.1780] [INSPIRE].

[61] A. Ficnar, S.S. Gubser and M. Gyulassy, Shooting String Holography of Jet Quenching at RHIC and $L H C$, arXiv:1311.6160 [INSPIRE].

[62] A. Ficnar, S.S. Gubser and M. Gyulassy, Holographic light quark jet quenching at RHIC and LHC via the shooting strings, arXiv:1404.0935 [INSPIRE].

[63] M. Mia, F. Chen, K. Dasgupta, P. Franche and S. Vaidya, Non-Extremality, Chemical Potential and the Infrared limit of Large-N Thermal QCD, Phys. Rev. D 86 (2012) 086002 [arXiv: 1202.5321] [INSPIRE]. 